\title{
Frustrated spin- $1 / 2$ Heisenberg magnet on a square-lattice bilayer: High-order study of the quantum critical behavior of the J1-J2-J1 model
}

DOI:

10.1103/PhysRevB.100.024401

\section{Document Version}

Final published version

Link to publication record in Manchester Research Explorer

Citation for published version (APA):

Bishop, R. F., Li, P. H. Y., Götze, O., \& Richter, J. (2019). Frustrated spin-1/2 Heisenberg magnet on a squarelattice bilayer: High-order study of the quantum critical behavior of the $J_{1}-J_{2}-J_{1}$ model. Physical Review $B, 100$, [024401 (14pp)]. https://doi.org/10.1103/PhysRevB.100.024401

\section{Published in:}

Physical Review B

\section{Citing this paper}

Please note that where the full-text provided on Manchester Research Explorer is the Author Accepted Manuscript or Proof version this may differ from the final Published version. If citing, it is advised that you check and use the publisher's definitive version.

\section{General rights}

Copyright and moral rights for the publications made accessible in the Research Explorer are retained by the authors and/or other copyright owners and it is a condition of accessing publications that users recognise and abide by the legal requirements associated with these rights.

\section{Takedown policy}

If you believe that this document breaches copyright please refer to the University of Manchester's Takedown Procedures [http://man.ac.uk/04Y6Bo] or contact uml.scholarlycommunications@manchester.ac.uk providing relevant details, so we can investigate your claim.

\section{OPEN ACCESS}




\title{
Frustrated spin- $\frac{1}{2}$ Heisenberg magnet on a square-lattice bilayer: High-order study of the quantum critical behavior of the $J_{1}-J_{2}-J_{1}^{\perp}$ model
}

\author{
R. F. Bishop,,$^{1,2, *}$ P. H. Y. Li, ${ }^{1,2, \dagger}$ O. Götze, ${ }^{3, \ddagger}$ and J. Richter ${ }^{3,4, \S}$ \\ ${ }^{1}$ School of Physics and Astronomy, Schuster Building, The University of Manchester, Manchester, M13 9PL, United Kingdom \\ ${ }^{2}$ School of Physics and Astronomy, University of Minnesota, 116 Church Street SE, Minneapolis, Minnesota 55455, USA \\ ${ }^{3}$ Institut für Theoretische Physik, Otto-von-Guericke Universität Magdeburg, P.O.Box 4120, 39016 Magdeburg, Germany \\ ${ }^{4}$ Max-Planck-Institut für Physik Komphexer Systeme, Nöthnitzer Straße 38, 01187 Dresden, Germany
}

(Received 9 May 2019; published 1 July 2019)

\begin{abstract}
The zero-temperature phase diagram of the spin- $\frac{1}{2} J_{1}-J_{2}-J_{1}^{\perp}$ model on an $A A$-stacked square-lattice bilayer is studied using the coupled cluster method implemented to very high orders. Both nearest-neighbor (NN) and frustrating next-nearest-neighbor Heisenberg exchange interactions, of strengths $J_{1}>0$ and $J_{2} \equiv \kappa J_{1}>0$, respectively, are included in each layer. The two layers are coupled via a NN interlayer Heisenberg exchange interaction with a strength $J_{1}^{\perp} \equiv \delta J_{1}$. The magnetic order parameter $M$ (viz., the sublattice magnetization) is calculated directly in the thermodynamic (infinite-lattice) limit for the two cases when both layers have antiferromagnetic ordering of either the Néel or the striped kind, and with the layers coupled so that NN spins between them are either parallel (when $\delta<0$ ) or antiparallel (when $\delta>0$ ) to one another. Calculations are performed at $n$th order in a well-defined sequence of approximations, which exactly preserve both the Goldstone linked-cluster theorem and the Hellmann-Feynman theorem, with $n \leqslant 10$. The sole approximation made is to extrapolate such sequences of $n$ th-order results for $M$ to the exact limit $n \rightarrow \infty$. By thus locating the points where $M$ vanishes, we calculate the full phase boundaries of the two collinear AFM phases in the $\kappa-\delta$ half-plane with $\kappa>0$. In particular, we provide the accurate estimate $(\kappa \approx 0.547, \delta \approx-0.45)$ for the position of the quantum triple point (QTP) in the region $\delta<0$. We also show that there is no counterpart of such a QTP in the region $\delta>0$, where the two quasiclassical phase boundaries show instead an "avoided crossing" behavior, such that the entire region that contains the nonclassical paramagnetic phases is singly connected.
\end{abstract}

DOI: 10.1103/PhysRevB.100.024401

\section{INTRODUCTION}

The frustrated spin- $\frac{1}{2} \quad J_{1}-J_{2}$ Heisenberg antiferromagnet on the square lattice, which contains isotropic Heisenberg exchange interactions with strengths $J_{1}>0$ between all nearest-neighbor (NN) pairs of spins and $J_{2}>0$ between all next-nearest-neighbor (NNN) pairs, has become a paradigmatic model of quantum magnetism. It has received enormous attention over the last 30 or so years [1-56], starting with its proposed relationship to the disappearance of antiferromagnetic (AFM) long-range order (LRO) in the high- $T_{c}$ cuprate superconductors. The conjecture here was that frustrated AFM exchange couplings might lead to a quantum spin liquid (QSL) state in which preformed pairs, or resonating valence bonds, could become superconducting upon doping $[57,58]$. More recently, as frustrated quantum magnets have emerged as an active research field in their own right, the model has become recognized as one of the most challenging quantum spin-lattice systems. Accordingly, it has been widely studied [1-56], by a large number of theoretical techniques, as a prototypical system in which to examine quantum phase transition

\footnotetext{
*raymond.bishop@manchester.ac.uk

†peggyhyli@gmail.com

¥oliver.goetze@ovgu.de

§johannes.richter@physik.uni-magdeburg.de
}

(QPTs) between quasiclassical ground-state (GS) phases with magnetic LRO and magnetically disordered (paramagnetic) quantum phases that are driven by frustration.

In addition to this extensive theoretical interest, it is also worth noting that several good experimental realizations of spin- $\frac{1}{2} J_{1}-J_{2}$ models on a quasi-two-dimensional square lattice exist with $J_{1}>0$ and $J_{2}>0$. Examples include the vanadium-layered oxide materials $\mathrm{Li}_{2} \mathrm{VO}(\mathrm{Si}, \mathrm{Ge}) \mathrm{O}_{4}$ [59] and the $B$-site ordered double-perovskite oxides $\mathrm{Ba}_{2} \mathrm{CuWO}_{6}$ [60], $\mathrm{Sr}_{2} \mathrm{CuMoO}_{6}$ [61], $\mathrm{Sr}_{2} \mathrm{CuWO}_{6}$ [61,62], and $\mathrm{Sr}_{2} \mathrm{CuTeO}_{6}$ [63].

Despite the intense interest in this model from both theorists and experimentalists, as outlined above, the nature of its GS phase around the value $\kappa=\frac{1}{2}$ of the frustration parameter $\kappa \equiv J_{2} / J_{1}$, which represents the point of maximum frustration in the classical version of the model, still remains largely unresolved. Thus, if the spins on the square-lattice sites carry spin quantum number $s$, the model becomes classical in the limit $s \rightarrow \infty$. In this classical limit the GS phase is simple in the two limiting cases $\kappa=0$ and $\kappa \rightarrow \infty$. Clearly, when $\kappa=0$, the model has Néel AFM order [i.e., with a magnetic wave vector $\mathbf{Q}=(\pi, \pi)$ ]. For nonzero values of $\kappa$ the Néel-ordered state has a GS energy per spin given by $E_{\mathrm{cl}} / N=2 J_{1}(-1+$ $\kappa) s^{2}$. By contrast, when $\kappa \rightarrow \infty$, the classical ordering is such that on each of the two equivalent interpenetrating sublattices (i.e., which comprise NNN sites on the original lattice connected by $J_{2}$ bonds) the spins are separately Néel ordered, and 
with a relative angle $\theta$ between the ordering directions on the two sublattices. The GS energy per spin in this case is given by $E_{\mathrm{cl}} / N=-2 J_{1} \kappa s^{2}$, independent of $\theta$, for any value of $\kappa$.

Clearly, the classical $J_{1}-J_{2}$ model on the square lattice thus has a first-order phase transition at $\kappa=\kappa_{\mathrm{cl}}=\frac{1}{2}$ between two AFM states, viz., the Néel state for $\kappa<\frac{1}{2}$ and an infinitely degenerate family of ground states specified by the relative angle $\theta$ between the ordering directions on the two interpenetrating sublattices, for $\kappa>\frac{1}{2}$. Thus, for $\kappa>\frac{1}{2}$, the classical GS manifold has $\mathrm{SU}(2) \times \mathrm{SU}(2)$ symmetry, which is larger than the SU(2) symmetry of the Hamiltonian. In this latter case, although the effects of the exchange fields $\left(J_{1}\right)$ between the two sublattices cancel out, the zero-point quantum fluctuations, as well as the thermal fluctuations, will depend on the angle $\theta$ between the two sublattice spin orientations. This leads to a prototypical example [64] of the phenomenon of order by disorder $[65,66]$, whereby the GS degeneracy is lifted by quantum fluctuations with the angle $\theta$ now selected to be 0 or $\pi$. The AFM GS ordering is now collinear, and the corresponding GS phase is a striped one consisting of successive alternating columns (or rows) of parallel spins [i.e., with a magnetic wave vector $\mathbf{Q}=(\pi, 0)$ or $\mathbf{Q}=(0, \pi)$, respectively]. The GS symmetry is thereby reduced from $\mathrm{SU}(2) \times \mathrm{SU}(2)$ to $\mathrm{SU}(2) \times Z_{2}$, and the collinear striped state breaks the invariance of the Heisenberg Hamiltonian under both spin rotations $[\mathrm{SU}(2)]$ and rotations by $90^{\circ}$ of the square lattice $\left(Z_{2}\right)$.

In the classical, $s \rightarrow \infty$, limit of the model, lowest-order spin-wave theory, wherein the effects of quantum fluctuations are taken into account perturbatively at $O\left(s^{-1}\right)$, thus shows [1] that the critical coupling $\kappa_{\mathrm{cl}}=\frac{1}{2}$ marks a first-order transition between the Néel and striped collinear AFM phases. In the extreme quantum case $s=\frac{1}{2}$, in which we are interested here, where quantum fluctuations now have to be taken fully into account beyond perturbation theory, it may be anticipated that these two quasiclassical AFM phases persist, but are now separated by one or more intermediate paramagnetic phases with no classical counterparts (i.e., without magnetic LRO). While there is essentially complete consensus that this scenario is realized in the spin- $\frac{1}{2} J_{1}-J_{2}$ model on the square lattice, the nature of both the phase (or phases) in the intermediate regime and their associated QPTs, as well as the precise critical values of $\kappa$ at which the latter occur, are still not completely resolved, despite many calculations over the last 30 or so years. These have included investigations of the model using a wide diversity of modern theoretical techniques and numerical tools of ever increasing sophistication. Examples include those based on mean-field theories of various (e.g., cluster, hierarchical) types $[3,28,32,46]$, the exact diagonalization (ED) of finite-sized clusters [2,9,10,17,20,24,31,35], linked-cluster series expansions $[3,11,15,22,33]$, the bondoperator formalism $[4,12,43]$, resonating valence bond (RVB) approaches [18,38-41,47,48], variational Monte Carlo (VMC) approaches based on various families of trial GS wave functions (e.g., RVB states, entangled plaquette states) [18,37-41,47-49,54], various quantum fieldtheoretical approaches [19,21,30,64] including the dynamic functional renormalization group [32,33], the density-matrix renormalization group (DMRG) [36,42,55], matrix-product or tensor-network approaches [29,34,39,51-53,56], and the coupled cluster method (CCM) [23,25-27,33,35,50].

While, in the intermediate region, where the GS phase or phases are nonmagnetic, the $\mathrm{SU}(2)$ spin symmetry is not broken, various symmetries of the lattice still may or may not be broken. In the former case, one can have various valencebond crystalline (VBC) phases where the lattice symmetries are broken by the formation of some pattern of spin singlets. Examples include the columnar dimer VBC phase, which breaks both translational and rotational lattice symmetries, and the plaquette VBC phase, which breaks only the translational symmetry. Alternatively, one could have a QSL phase that conserves all lattice symmetries. Such a QSL phase could be either gapped or gapless (e.g., of the $Z_{2}$ type).

Each of these phases has been proposed to form the stable GS in part of all of the paramagnetic intermediate regime of the spin- $\frac{1}{2} J_{1}-J_{2}$ model on the square lattice by various of the above-cited references, with no overall consensus having yet emerged. Part of the reason for this uncertainty undoubtedly must lie in the fact that of the various methods discussed above that have high potential accuracy and/or are capable of systematic improvement via some well-defined hierarchical approximation scheme, almost all are either intrinsically biased in favor of some particular GS phase and/or are not directly performed in the thermodynamic (infinite-lattice) limit of interest. In the latter regard, for example, the great majority of the techniques employed are performed on lattices of a finite size ( $N$ spins), and some form of finite-size scaling is then used to extrapolate to the thermodynamic $(N \rightarrow \infty)$ limit.

As has been very rigorously and authoritatively demonstrated in a recent study [67] of the spin- $\frac{1}{2} J-Q$ model on the square lattice, for which the infamous quantum Monte Carlo (QMC) minus-sign problem is absent, and hence where large-scale QMC calculations can be undertaken, by contrast with the corresponding $J_{1}-J_{2}$ model of interest here, such extrapolations to the thermodynamic limit can have great uncertainties. This is specially true in cases where is little or no analytic guidance from theoretical considerations, as is often the case, but can also even hold when such guidance is present. In this context it is particularly noteworthy that the CCM [68-85] provides a rather singular example of a theoretical quantum many-body technique that can and does study arbitrary spin-lattice models directly in the thermodynamic limit. It is precisely for that reason that we employ it here.

Furthermore, in view of the still puzzling nature of the phase or phases present in the intermediate paramagnetic regime of the spin- $\frac{1}{2} J_{1}-J_{2}$ model on the square lattice around the value $\kappa=\frac{1}{2}$ of the frustration parameter, it is also potentially useful to examine a larger class of systems for which this model reduces to a special case. Thus, we are strongly motivated to consider the corresponding spin- $\frac{1}{2} J_{1}-J_{2}-J_{1}^{\perp}$ model on a square-lattice bilayer. Each of the two monolayers is just a frustrated $J_{1}-J_{2}$ system, but the two layers are now connected by Heisenberg exchange bonds of strength $J_{1}^{\perp} \equiv \delta J_{1}$ between $\mathrm{NN}$ interlayer pairs of spins, with the two layers arranged in $A A$ stacking [i.e., with each site of one (horizontal) monolayer placed immediately above its counterpart on the other monolayer]. The original $J_{1}-J_{2}$ model is then just the special case 
$\delta=0$ of the larger $J_{1}-J_{2}-J_{1}^{\perp}$ model. In this paper we use the CCM to study the spin- $-\frac{1}{2} J_{1}-J_{2}-J_{1}^{\perp}$ model on a square-lattice bilayer, for both signs of the interlayer coupling parameter $\delta$. In particular, we will concentrate our efforts on examining the complete phase boundaries of the two quasiclassical collinear (AFM) phases (viz., the phases with Néel and striped AFM order on each of the coupled monolayers) in the $\kappa-\delta$ half-plane with $\kappa>0$ (and $J_{1}>0$ ), and specifically in the window $0 \leqslant$ $\kappa \leqslant 1$ that contains the intermediate paramagnetic regime in the case $\delta=0$.

In this context it is interesting to note too that the spin$\frac{1}{2} J_{1}-J_{2}-J_{1}^{\perp}$ model has also been previously studied on a stacked square lattice (i.e., where the number of layers $n \rightarrow \infty$, rather than the case $n=2$ studied here) [23], where use was also made of the CCM. Thus, the bilayer model we study here lies, in some sense, between the strictly twodimensional square-lattice $J_{1}-J_{2}$ model (i.e., where $\delta=0$ ) and the strictly three-dimensional $J_{1}-J_{2}-J_{1}^{\perp}$ model on the stacked square lattice with an infinite number of layers. Furthermore, unlike the latter case, the bilayer case also exhibits the additional physical phenomenon of dimerization between $\mathrm{NN}$ interlayer pairs, as discussed more fully in Sec. II. These features thus provide considerable additional motivation to study the bilayer model.

The plan for the remainder of this paper is as follows. The $J_{1}-J_{2}-J_{1}^{\perp}$ model is itself first described in Sec. II, where we also discuss more fully the main features of the limiting case $J_{1}^{\perp}=0$ of the monolayer model. We also give there some discussion of what we might expect to be some of the main features of the phase boundaries of the two quasiclassical AFM phases as the interlayer coupling parameter $\delta \equiv J_{1}^{\perp} / J_{1}$ is introduced. The main features of the CCM as applied to quantum spin-lattice problems are then reviewed in Sec. III before our numerical results are presented in Sec. IV. Finally, our findings are summarized and discussed in Sec. V, where we also make comparisons with the results of others.

\section{MODEL}

The Hamiltonian of the $J_{1}-J_{2}-J_{1}^{\perp}$ model on a square-lattice bilayer is specified as

$$
\begin{aligned}
H= & J_{1} \sum_{\langle i, j\rangle, \alpha} \mathbf{s}_{i, \alpha} \cdot \mathbf{s}_{j, \alpha}+J_{2} \sum_{\langle\langle i, k\rangle\rangle, \alpha} \mathbf{s}_{i, \alpha} \cdot \mathbf{s}_{k, \alpha} \\
& +J_{1}^{\perp} \sum_{i} \mathbf{s}_{i, 1} \cdot \mathbf{s}_{i, 2} \\
\equiv & J_{1} h(\kappa, \delta) ; \quad \kappa \equiv J_{2} / J_{1}, \quad \delta \equiv J_{1}^{\perp} / J_{1},
\end{aligned}
$$

such that the sites on each (horizontal) monolayer are labeled by the index $i$ (i.e., with the two layers in $A A$ stacking such that sites $i$ on the top layer lie vertically above those on the bottom layer), and the two layers are labeled by the index $\alpha=1,2$. Every site $(i, \alpha)$ is occupied by a spin- $s$ particle described in terms of the usual SU(2) operators $\mathbf{s}_{i, \alpha} \equiv\left(s_{i, \alpha}^{x}, s_{i, \alpha}^{y}, s_{i, \alpha}^{z}\right)$, with $\mathbf{s}_{i, \alpha}^{2}=s(s+1) \mathbb{1}$, and where we restrict discussion here to the case $s=\frac{1}{2}$. The first two sums over $\langle i, j\rangle$ and $\langle\langle i, k\rangle\rangle$ in Eq. (1) run over all NN and NNN intralayer pairs of spins, respectively, with each Heisenberg bond (with respective strengths
$J_{1}$ and $J_{2}$ ) counted once and once only. The third sum in Eq. (1) over the index $i$ counts all corresponding interlayer NN Heisenberg bonds of strength $J_{1}^{\perp}$. We shall be interested here in the case when both intralayer bonds are AFM in nature (i.e., $J_{1}>0$ and $J_{2} \equiv \kappa J_{1}>0$ ), such that frustration is present in each monolayer, but where the interlayer coupling parameter $J_{1}^{\perp} \equiv \delta J_{1}$ may be either AFM $(\delta>0)$ or ferromagnetic (FM) $(\delta<0)$ in nature. Since the parameter $J_{1}$ merely sets the overall energy scale, the Hamiltonian may be expressed as in the last line of Eq. (1), such that the relevant parameters of the model are $\kappa$ and $\delta$.

Our main interest here will thus be to investigate the regions of stability of the two collinear AFM phases in each monolayer (i.e., the quasiclassical Néel and striped phases) in the $\kappa-\delta$ half-plane with $\kappa>0$, as the interlayer coupling $\delta$ is turned on. The square-lattice bilayer is illustrated in Fig. 1(a), while the patterns of spins of the two quasiclassical AFM phases on each monolayer are shown in Figs. 1(b) and 1(c), respectively. For both AFM phases the original square lattice (with spacing $d$ ) is decomposed into two equivalent sublattices. For the Néel state each sublattice is itself square (i.e., with spacing $\sqrt{2} d \times \sqrt{2} d$ ), such that each site on one sublattice has its $4 \mathrm{NN}$ sites on the original lattice on the other sublattice. By contrast, for the striped states, the original lattice is decomposed into equivalent sublattices chosen either as alternating columns (each with spacing $2 d \times d$ ) or as alternating rows (each with spacing $d \times 2 d$ ). Each of the corresponding classical AFM phases then has its spins on one sublattice pointing in a given, arbitrary (say, down) direction, and those on the other sublattice pointing in the opposite (say, up) direction. The classical Néel state is thus as shown in Fig. 1(b), while the classical columnar striped state is as shown in Fig. 1(c).

For the case of the spin- $\frac{1}{2}$ square-lattice monolayer $(\delta=0)$ there is essentially complete agreement that Néel order persists for $\kappa<\kappa_{c_{1}}$, striped order persists for $\kappa>\kappa_{c_{2}}$, and some paramagnetic phase (or phases) form the stable GS phase in the intermediate regime $\kappa_{c_{1}}<\kappa<\kappa_{c_{2}}$. While the nature of the phase(s) in the intermediate regime remains unresolved, as noted in Sec. I, modern high-quality calculations do seem to be converging on values for the two critical points of $\kappa_{c_{1}} \approx 0.43(3)$ and $\kappa_{c_{2}} \approx 0.605(15)$.

Thus, for example, three recent independent DMRG calculations yielded the values $\kappa_{c_{1}} \approx 0.41, \kappa_{c_{2}} \approx 0.62$ [36], $\kappa_{c_{1}} \approx$ $0.44, \kappa_{c_{2}} \approx 0.61$ [42], and $\kappa_{c_{1}} \approx 0.46, \kappa_{c_{2}} \approx 0.62$ [55], while two high-order CCM calculations yielded the values $\kappa_{c_{1}} \approx$ $0.447, \kappa_{c_{2}} \approx 0.586$ [35] and $\kappa_{c_{1}} \approx 0.454, \kappa_{c_{2}} \approx 0.588$ [50]. Similar results have also been found, for example, from a plaquette-renormalized tensor-network study [34] that gave values $\kappa_{c_{1}} \approx 0.40, \kappa_{c_{2}} \approx 0.62$; a renormalization group $(\mathrm{RG})$ approach [45], in which the RG flows were numerically integrated, that gave values $\kappa_{c_{1}} \approx 0.416, \kappa_{c_{2}} \approx 0.606$; a cluster mean-field theory approach [46] that gave values $\kappa_{c_{1}} \approx 0.42$, $\kappa_{c_{2}} \approx 0.59$; a VMC calculation using an AFM fermionic RVB class of trial wave functions [48] that gave values $\kappa_{c_{1}} \approx 0.45$, $\kappa_{c_{2}} \approx 0.6$; and a separate many-variable VMC calculation combined with quantum-number projections [49] that gave values $\kappa_{c_{1}} \approx 0.4, \kappa_{c_{2}} \approx 0.6$.

We should note, however, that while there is broad agreement on the value for $\kappa_{c_{2}}$, there are still outlier calculations 


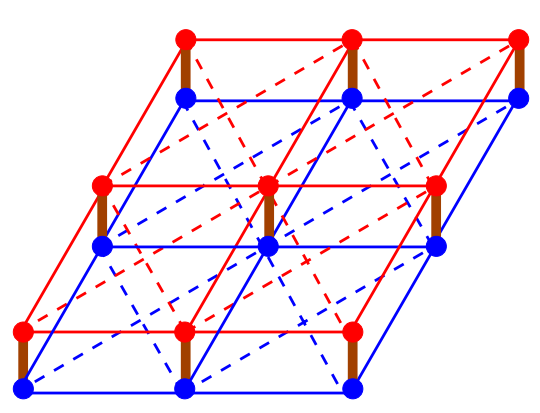

(a)

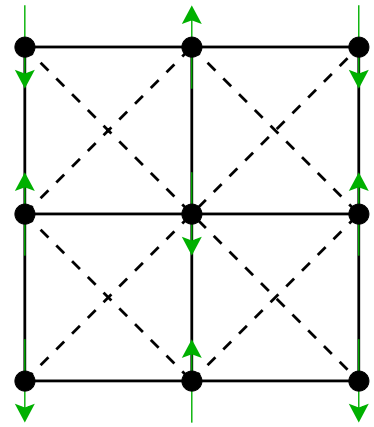

(b)

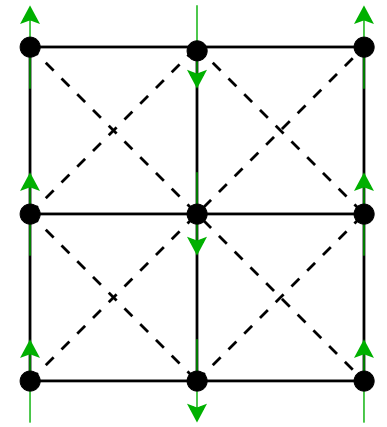

(c)

FIG. 1. The $J_{1}-J_{2}-J_{1}^{\perp}$ model on the square-lattice bilayer, showing (a) the two layers 1 (red) and 2 (blue), the nearest-neighbor intralayer $J_{1}$ bonds as thin (red or blue) solid lines; the nearest-neighbor interlayer $J_{1}^{\perp}$ bonds as thick (brown) solid lines, and the next-nearest-neighbor intralayer $J_{2}$ bonds as dashed (red or blue) lines; (b) the monolayer Néel state; and (c) the monolayer columnar striped state. Lattice sites are shown by filled circles and spins by the (green) arrows.

for $\kappa_{c_{1}}$. For example, a bond-operator formalism approach that included cubic and quartic interactions beyond the harmonic approximation [43] yielded a lower value of $\kappa_{c_{1}} \approx 0.34$ (and $\kappa_{c_{2}} \approx 0.59$ ), while a recent approach using the cluster update algorithm for tensor product states [51] yielded the much higher value of $\kappa_{c_{1}} \approx 0.572$. In this context it is interesting to note too that a large-scale ED calculation using finite-size scaling to the thermodynamic limit $(N \rightarrow \infty)$ on finite square lattices of up to $N=40$ sites [31] yielded values $\kappa_{c_{1}} \approx 0.35$, $\kappa_{c_{2}} \approx 0.66$ based on the points where the Néel and striped order parameters vanish, respectively, but also gave values $\kappa_{c_{1}} \approx 0.46, \kappa_{c_{2}} \approx 0.60$ based on points where the respective zero-field transverse (uniform) magnetic susceptibility vanishes. The latter estimates are clearly in much better agreement with the modern consensual values. On the other hand, we should note that the vanishing of the magnetic susceptibility only denotes the opening up of a new gapped phase. Any nonmagnetic gapless state (e.g., of the QSL variety) would not, of course, be seen by calculations of the susceptibility alone.

Turning our attention now to the bilayer, it is clear that the interlayer $J_{1}^{\perp}$ bonds have no additional frustrating effect on the intralayer magnetic LRO. Indeed, in the classical limit $(s \rightarrow \infty)$ they have zero effect. However, for finite spin quantum numbers $s$, if we consider first the case of zero frustration $(\kappa=0)$, the $J_{1}$ and $J_{1}^{\perp}$ bonds do still compete with one another since the $J_{1}^{\perp}$ bonds by themselves promote the formation of NN interlayer dimers. For the present spin- $\frac{1}{2}$ case when $J_{1}^{\perp}>0$ these are spin-singlet pairs, while for $J_{1}^{\perp}<0$ they are spin-triplet pairs. Thus, even with zero frustration $\left(J_{2}=0\right)$, the introduction of AFM $J_{1}^{\perp}$ bonds induces a competition between a GS magnetic phase with Néel LRO and a nonclassical paramagnetic phase of the VBC kind, which is formed of interlayer dimers. The resulting spin- $\frac{1}{2} J_{1}-J_{1}^{\perp}$ model on a square-lattice bilayer has been studied previously [86-102]. Since QMC calculations can be performed in this case (i.e., when $\kappa=0)$, the position $\delta_{c_{1}}^{>}(\kappa=0)$ of the QPT between the Néel-ordered state and the quantum disordered interlayer-dimer VBC (IDVBC) state can be ascertained with high accuracy. For example, a finite-size scaling of QMC results on lattices with $2 L^{2}$ spins with $L \leqslant 10$ [90] gave a value $\delta_{c_{1}}^{>}(\kappa=0)=2.51(2)$, while a more recent QMC calculation of Wang et al. [96] using the improved stochastic series-expansion algorithm with operator-loop updates and finite-size scaling on $L \times L \times 2$ lattices with $L \leqslant 42$ gave the very precise value $\delta_{c_{1}}^{>}(\kappa=0)=2.5220(1)$. An exponentbiased SE analysis of Zheng [93] gave the comparable result $\delta_{c_{1}}^{>}(\kappa=0)=2.537(5)$.

We turn now finally to the case of interest here where we also introduce intralayer frustration via the NNN AFM $J_{2}$ bonds. The resulting spin- $\frac{1}{2} J_{1}-J_{2}-J_{1}^{\perp}$ model on a squarelattice bilayer has received much less attention $[103,104]$ than either of the limiting cases $\delta=0$ [1-56] or $\kappa=0$ [86-102] discussed above. We note, however, that a very recent paper [105] studied the case where frustration is introduced instead via an interlayer NNN AFM $J_{2}^{\perp}$ bond, resulting in a $J_{1}-J_{1}^{\perp}-J_{2}^{\perp}$ model, with very different properties and behavior (and see also Ref. [106]).

Before presenting our results in Sec. IV for the $J_{1}-J_{2}-J_{1}^{\perp}$ model it may be worthwhile to outline first our aims and expectations. As the interlayer coupling parameter $\delta$ is turned on, we anticipate that its initial effect at a fixed value of $\kappa$ at which magnetic order of either the Néel or striped sort exists will be to enhance the stability of the corresponding quasiclassical state since the effect of the $J_{1}^{\perp}$ bonds is to increase the number of NN bonds and hence to take a step toward three dimensionality. A priori, one can expect that this effect is roughly symmetric with respect to small positive and negative values of $\delta$. Hence, we anticipate that on a $\kappa \delta$ plot the Néel and striped phase boundaries will both show a cusp at $\delta=0$. Thus, we expect that $\kappa_{c_{1}}(\delta)$ will initially increase and $\kappa_{c_{2}}(\delta)$ will initially decrease as $\delta$ is either increased to small positive values or decreased to small negative values. Accordingly, we are particularly interested in what then happens as $|\delta|$ is increased further in both the FM $(\delta<0)$ and AFM $(\delta>0)$ regimes of interlayer coupling. The situation is expected to be very different in the two cases.

Thus, first, in the region where $\delta<0$, it is evident that in the limit $\delta \rightarrow-\infty$ the system will simply behave as a spin-1 $J_{1}-J_{2}$ model on a square-lattice monolayer. Unlike the corresponding spin- $\frac{1}{2}$ case, the spin- $1 J_{1}-J_{2}$ model on the square lattice seems to show [107-109] a direct transition 
between the Néel and striped phases at a critical value $\kappa \approx$ 0.55 , although an early DMRG calculation [110] indicated a disordered paramagnetic phase in the narrow region $0.525 \lesssim$ $\kappa \lesssim 0.555$. Interestingly, a later and very recent DMRG calculation [109] using larger finite lattices showed that if such an intermediate region did exist, it could do so only in the much smaller regime $0.545 \lesssim \kappa \lesssim 0.550$. While the system sizes in the DMRG calculations $[109,110]$ were too small for a critical analysis, both the CCM analysis $[107,108]$ and an infinite projected entangled-pair-state analysis [109], have shown that the direct transition between the Néel and striped phases for the spin- 1 case is a first-order transition. The best estimate for the critical coupling of the transition is $\kappa \approx 0.549$ [109].

Returning to our bilayer model, let us denote by $\delta_{c_{1}}^{\mathrm{F}}(\kappa)$ and $\delta_{c_{2}}^{\mathrm{F}}(\kappa)$ the critical values of $\delta$ (for a given value of $\kappa$ ) at which Néel order and striped order, respectively, melt in the regime of FM interlayer coupling $(\delta<0)$. Equivalently, these phase boundaries $\delta=\delta_{c_{1}}^{\mathrm{F}}(\kappa)$ and $\delta=\delta_{c_{2}}^{\mathrm{F}}(\kappa)$ are also denoted, respectively, as $\kappa=\kappa_{c_{1}}^{\mathrm{F}}(\delta)$ and $\kappa=\kappa_{c_{2}}^{\mathrm{F}}(\delta)$. In the light of the above discussion it seems clear that in the half-plane $\delta<0$ there must exist a quantum triple point (QTP) that occurs at a value $\delta=\delta_{\mathrm{T}}^{\mathrm{F}}$ such that $\kappa_{c_{1}}^{\mathrm{F}}\left(\delta_{\mathrm{T}}^{\mathrm{F}}\right)=\kappa_{c_{2}}^{\mathrm{F}}\left(\delta_{\mathrm{T}}^{\mathrm{F}}\right)$ or, equivalently, when $\delta_{c_{1}}^{\mathrm{F}}\left(\kappa_{\mathrm{T}}^{\mathrm{F}}\right)=\delta_{c_{2}}^{\mathrm{F}}\left(\kappa_{\mathrm{T}}^{\mathrm{F}}\right)$. Thus, if the position of this QTP is $\left(\kappa_{\mathrm{T}}^{\mathrm{F}}, \delta_{\mathrm{T}}^{\mathrm{F}}\right)$, then for all values $\delta<\delta_{\mathrm{T}}^{\mathrm{F}}$ there will be a direct transition between the Néel and striped phases at a value $\kappa^{\mathrm{F}}(\delta)$, where we expect $\lim _{\delta \rightarrow-\infty} \kappa^{\mathrm{F}}(\delta) \approx 0.549$. One of our aims will be to evaluate accurately the position $\left(\kappa_{\mathrm{T}}^{\mathrm{F}}, \delta_{\mathrm{T}}^{\mathrm{F}}\right)$ of the QTP where the Néel, striped, and disordered paramagnetic phases meet in the half-plane $\delta<0$. It seems almost certain that $\kappa_{\mathrm{T}}^{\mathrm{F}}$ will lie between the monolayer values $\kappa_{c_{1}}(\delta=0) \approx$ $0.43(3)$ and $\kappa_{c_{2}}(\delta=0) \approx 0.605(15)$.

The possible scenarios in the half-plane $\delta>0$ are even more interesting. One possibility is the obvious analog to that discussed above, with another QTP between the Néel, striped, and intermediate paramagnetic phases. However, in this scenario, such a QTP would presumably have to be accompanied by another QTP, at a larger value of $\delta$, now between the Néel and striped phases together with the gapped IDVBC state that we know must physically occur for large enough values of $\delta$ at any fixed value of $\kappa$. Such a scenario (at least as far as the first QTP is concerned) was obtained in an earlier CCM calculation [23] of the spin- $\frac{1}{2} J_{1}-J_{2}-J_{1}^{\perp}$ model on the stacked square lattice (i.e., the same model as considered here but with an infinite number of layers in which all NN interlayer pairs are connected via $J_{1}^{\perp}$ bonds). In this case, of course, no IDVBC state occurs, and the Néel and striped AFM phases simply undergo a first-order transition for all values of the coupling parameter $\delta$ beyond the first (and only) QTP in this case.

An alternative, perhaps more intriguing, scenario in the half-plane $\delta>0$ is one in which the boundaries of the two quasiclassical AFM phases turn back on themselves, in a reentrant fashion, sufficiently rapidly as $\delta$ is increased so that they avoid crossing. One of our major aims here is to perform sufficiently accurate calculations as to be able to distinguish with confidence between such different scenarios. Before we present our findings in Sec. IV, however, we first briefly discuss in Sec. III the most important features of the CCM that we use to obtain them.

\section{COUPLED CLUSTER METHOD}

The CCM [68-85] is widely recognized as providing one of the most flexible, most widely utilized, and most accurate of all available $a b$ initio techniques in modern microscopic quantum many-body theory. One of the keys to its success is the fact that it preserves size-extensivity and size-consistency at every level of approximation, thereby enabling it to be implemented from the very outset in the thermodynamic $(N \rightarrow$ $\infty)$ limit. Hence, any errors associated with finite-size scaling, as needs to be performed in almost all competing methods, are obviated. A second key to the success of the CCM lies in the fact that it also exactly preserves at all levels of approximation the very important Hellmann-Feynman theorem as well as the Goldstone linked-cluster theorem. A third key to its success is that there exist well-defined, systematic, and very widely tested hierarchies of truncations within which the method can be computationally implemented to very high orders of approximation, as will be done here. Since the CCM becomes exact within such a truncation hierarchy as the order $n$ of the approximation tends to infinity $(n \rightarrow \infty)$, the only approximation ever made is in the extrapolation of such a sequence of approximants for any physical parameter calculated for the system under study. The combination of these features ensures that the CCM yields accurate and self-consistent sets of results for all GS and excited-state (ES) quantities calculated.

Amongst many applications to quantum many-body problems in fields as diverse as nuclear physics, subnuclear physics, quantum chemistry, atomic and molecular physics, quantum optics, and condensed matter physics, the CCM has, in particular, by now been applied to a wide variety of spin-lattice systems of interest in quantum magnetism (see, e.g., Refs. $[14,23,25-27,33,35,50,82,83,85,107,108,111]$ and references therein). Since its application to such systems has already been widely described in the literature, therefore, we content ourselves here with presenting a brief overview of only those features that are most relevant to us now.

The first step in any implementation of the CCM is to choose a suitable model (or reference) state $|\Phi\rangle$ for the $N$ body system with $(N \rightarrow \infty)$ under consideration, together with a complete set of mutually commuting, multiconfigurational creation operators $C_{I}^{+} \equiv\left(C_{I}^{-}\right)^{\dagger}$. The main requirement on $|\Phi\rangle$ is that it should be a cyclic vector (or, equivalently, a generalized vacuum state) with respect to the set of operators $\left\{C_{I}^{+}\right\}$. The set-index $I$ here is used to indicate a complete labeling of the many-particle configuration created in the state $C_{I}^{+}|\Phi\rangle$. We thus require the set $\left\{|\Phi\rangle ; C_{I}^{+}\right\}$to obey the conditions

$$
\begin{gathered}
\sum_{I} C_{I}^{+}|\Phi\rangle\langle\Phi| C_{I}^{-}=\mathbb{1}, \\
\left\langle\Phi\left|C_{I}^{+}=0=C_{I}^{-}\right| \Phi\right\rangle, \quad \forall I \neq 0 ; \quad C_{0}^{+} \equiv \mathbb{1}, \\
{\left[C_{I}^{+}, C_{J}^{+}\right]=0=\left[C_{I}^{-}, C_{J}^{-}\right],}
\end{gathered}
$$

where $\mathbb{1}$ is the unit vector in the $N$-particle Hilbert space. It is also convenient to choose the states $\left\{C_{I}^{+}|\Phi\rangle\right\}$ that so span the $\mathrm{N}$-body Hilbert space to be an orthonormal set

$$
\left\langle\Phi\left|C_{I}^{-} C_{J}^{+}\right| \Phi\right\rangle=\delta_{I, J},
$$

with $\delta_{I, J}$ a suitably generalized Kronecker symbol. 
The exact many-body GS ket and bra states $|\Psi\rangle$ and $\langle\tilde{\Psi}|$ $(=\langle\Psi| /\langle\Psi \mid \Psi\rangle)$, respectively, which satisfy the respective GS Schrödinger equations

$$
H|\Psi\rangle=E|\Psi\rangle, \quad\langle\tilde{\Psi}| H=E\langle\tilde{\Psi}|,
$$

with $|\Psi\rangle$ now satisfying the intermediate normalization condition $\langle\Phi \mid \Psi\rangle=1=\langle\Phi \mid \Phi\rangle$, together with $\langle\tilde{\Psi} \mid \Psi\rangle=1$, are now parametrized within the CCM with respect to the model state $|\Phi\rangle$ via the distinctive exponentiated forms of correlation operators

$$
\begin{gathered}
|\Psi\rangle=e^{S}|\Phi\rangle, \quad S=\sum_{I \neq 0} \mathcal{S}_{I} C_{I}^{+}, \\
\langle\tilde{\Psi}|=\langle\Phi| \tilde{S} e^{-S}, \quad \tilde{S}=1+\sum_{I \neq 0} \tilde{\mathcal{S}}_{I} C_{I}^{-},
\end{gathered}
$$

that are one of the distinguishing features of the method. Although Hermiticity implies that the destruction correlation operator $\tilde{S}$ is formally related to its creation counterpart $S$ via the relation

$$
\langle\Phi| \tilde{S}=\frac{\langle\Phi| e^{S^{\dagger}} e^{S}}{\left\langle\Phi\left|e^{S^{\dagger}} e^{S}\right| \Phi\right\rangle},
$$

the CCM treats $S$ and $\tilde{S}$ as independent operators. They will clearly satisfy Eq. (9) when no approximations are made, but may violate it when truncations are made in the sums over the index $I$ in Eqs. (7) and (8), as described below in practical implementations. The compensation paid for this loss of explicit Hermiticity is the huge advantage that in all such truncations the Hellmann-Feynman theorem is now manifestly maintained.

All GS properties of the system may thus be calculated in terms of the set of real $c$-number correlation coefficients $\left\{\mathcal{S}_{I}, \tilde{\mathcal{S}}_{I}\right\}$. In turn, these may be found by insertion of the parametrizations of Eqs. (7) and (8) into the respective Schrödinger equations (6), followed by projection onto the complete sets of states $\langle\Phi| C_{I}^{-}$and $C_{I}^{+}|\Phi\rangle$, respectively. As a completely equivalent alternative procedure we may derive $\left\{\mathcal{S}_{I}, \tilde{\mathcal{S}}_{I}\right\}$ by demanding that the GS energy expectation value functional $\bar{H}=\bar{H}\left(\mathcal{S}_{I}, \tilde{\mathcal{S}}_{I}\right)$, defined as

$$
\bar{H} \equiv\langle\tilde{\Psi}|H| \Psi\rangle=\left\langle\Phi\left|\tilde{S} e^{-S} H e^{S}\right| \Phi\right\rangle,
$$

be an extremum with respect to the entire set of parameters $\left\{\mathcal{S}_{I}, \tilde{\mathcal{S}}_{I}\right\}$. By either method we may readily derive the sets of equations

$$
\begin{gathered}
\left\langle\Phi\left|C_{I}^{-} e^{-S} H e^{S}\right| \Phi\right\rangle=0, \quad \forall I \neq 0 ; \\
\langle\Phi| \tilde{S}\left(e^{-S}\left[H, C_{I}^{+}\right] e^{S}|\Phi\rangle=0, \quad \forall I \neq 0 .\right.
\end{gathered}
$$

By using Eq. (11) we may also show that the GS energy at the stationary point may be expressed purely in terms of the set of creation coefficients $\left\{\mathcal{S}_{I}\right\}$ as

$$
E=E\left(\mathcal{S}_{I}\right)=\left\langle\Phi\left|e^{-S} H e^{S}\right| \Phi\right\rangle .
$$

Correspondingly, Eq. (12) may be written in the equivalent form

$$
\left\langle\Phi\left|\tilde{S}\left(e^{-S} H e^{S}-E\right) C_{I}^{+}\right| \Phi\right\rangle=0, \quad \forall I \neq 0 .
$$

By contrast, the GS expectation value $\bar{A} \equiv\langle\tilde{\Psi}|A| \Psi\rangle$ of any other operator $A$ requires both sets of GS CCM coefficients for its evaluation

$$
\bar{A}=\bar{A}\left(\mathcal{S}_{I}, \tilde{\mathcal{S}}_{I}\right)=\left\langle\Phi\left|\tilde{S} e^{-S} A e^{S}\right| \Phi\right\rangle .
$$

We note that the characteristic CCM exponentiated operators $e^{ \pm S}$ only enter into Eqs. (11) and (12), which need to be solved for the GS coefficients $\left\{\mathcal{S}_{I}, \tilde{\mathcal{S}}_{I}\right\}$, in the form of the associated similarity transform of the Hamiltonian $e^{-S} H e^{S}$. In order to solve Eqs. (11) and (12) in practice we utilize the nested commutator expansion

$$
e^{-S} H e^{S}=\sum_{n=0}^{\infty} \frac{1}{n !}[H, S]_{n},
$$

where the $n$-fold nested commutators $[H, S]_{n}$ are defined iteratively as

$$
[H, S]_{n} \equiv\left[[H, S]_{n-1}, S\right] ; \quad[H, S]_{0}=H .
$$

Another key feature of the CCM parametrizations of Eqs. (7) and (8) is that the otherwise infinite sum in Eq. (16) now terminates in practice for all Hamiltonians that contain only finite-order multinomials in the appropriate singleparticle operators, as in the present case. The reason for this is simple, namely, that all components in the expansion of Eq. (7) mutually commute by construction, as in Eq. (4). For the present Hamiltonian of Eq. (1), which is bilinear in the basic one-body operators $\left(s_{i, \alpha}^{x}, s_{i, \alpha}^{y}, s_{i, \alpha}^{z}\right)$, the sum in Eq. (16) will terminate with the term $n=2$ for the choices for $\left\{|\Phi\rangle ; C_{I}^{+}\right\}$that we describe below, due to the basic $\mathrm{SU}(2)$ commutation relations. Thus, all nested commutators with $n>2$ simply vanish identically.

For the same reason, all terms in the expansion of $\bar{H}$ are linked, and it is this fact that leads to the CCM satisfying the Goldstone linked-cluster theorem (and consequently being size-extensive) at all levels of truncation in the expansions of Eqs. (7) and (8). Thus, in the solutions of Eqs. (11) and (12), the sole approximation made is in what set of configurations $\{I\}$ will be retained in the expansions of Eqs. (7) and (8), as described below.

We turn now to the choice of model state $|\Phi\rangle$ and the associated set of multiconfigurational creation operators $\left\{C_{I}^{+}\right\}$ for the present case. In any spin-lattice application of the CCM, a convenient (but not the only) choice for $|\Phi\rangle$ is always any quasiclassical state with perfect magnetic LRO, i.e., one for which the spin on every lattice site is specified independently via its given spin projection onto some specified spin quantization axis. Here, we will thus use both the Néel and striped AFM states as our independent CCM model states. It is highly convenient to treat all such states in the same way, so that all sites may be treated equivalently. A simple means of doing so is to choose a local spin quantization axis independently on each site (i.e., equivalently, by making a suitable passive rotation of each spin separately) so that in these local axes the reference state is a tensor product of spin-down states, $|\Phi\rangle=|\downarrow \downarrow \downarrow \ldots \downarrow\rangle$, such that all spins point along the negative $z_{s}$ direction in these local sets of axes. A beneficial effect of choosing such rotations is that all cases may henceforward be treated on an equal footing and by a universal computational code. The cases are distinguished 
only by that the spin Hamiltonian needs to be rewritten in terms of the particular local axes needed for each specific model state.

Such passive rotations are unitary transformations that leave the basic SU(2) algebra unchanged, but also have the other beneficial effect of allowing us to choose the operators $C_{I}^{+}$as products of single spin-raising operators $s_{k, \alpha}^{+} \equiv s_{k, \alpha}^{x}+$ $i s_{k, \alpha}^{y}$. Thus, we have that the set-index $I$ becomes a set of lattice indices $\{I\} \rightarrow\left\{l_{1}, l_{2}, \ldots, l_{n} ; n=1,2, \ldots, 2 s N\right\}$, where $l_{i} \equiv\left(k_{i}, \alpha\right)$, and in which any given lattice site index $l_{i}$ may be repeated so that it appears no more than $2 s$ times, where $s$ is the spin quantum number of the spins in the general case. Thus, we have $C_{I}^{+} \rightarrow s_{l_{1}}^{+} s_{l_{2}}^{+} \ldots s_{l_{n}}^{+}$, with $n=1,2, \ldots, 2 s N$. Here, we take $s=\frac{1}{2}$, so that in each configuration $I$ no lattice site may appear more than once.

In these local rotated spin axes the order parameter (i.e., the sublattice magnetization) takes the universal form

$$
M=-\frac{1}{N} \sum_{i=1}^{N}\left\langle\Phi\left|\tilde{S} e^{-S} s_{l_{i}}^{z} S^{S}\right| \Phi\right\rangle .
$$

Once again, the expression $e^{-S} S_{l_{i}}^{z} e^{S}$ may be evaluated exactly via a nested commutator expansion akin to Eq. (16) that now terminates at the term with $n=1$.

As we have indicated above, the only approximation that we make in implementing the CCM is the truncation of the expansion of Eqs. (7) and (8) for the correlation operators $S$ and $\tilde{S}$. We shall use here the well-established localized (lattice-animal-based subsystem) LSUB $n$ scheme wherein we retain at $n$th order all multispin-flip correlations on the lattice over no more than $n$ contiguous sites. A set of sites is contiguous in this sense if every site in the set is NN to at least one other in the set, in some specified geometry. As the truncation index $n$ tends to infinity $(n \rightarrow \infty)$, the corresponding LSUB $n$ approximation becomes exact.

One may use the space- and point-group symmetries of the lattice and the particular CCM model state $|\Phi\rangle$ being used, as well as any pertinent conservation laws, to reduce the number of independent configurations retained at any order $n$ of approximation. For example, for each of the model states considered in Sec. IV (i.e., the Néel and striped states on each monolayer), the Hamiltonian of Eq. (1) conserves the total $z$ component of spin, $s_{T}^{z} \equiv \sum_{i=1}^{N} s_{l_{i}}^{z}$ (where global spin axes are now assumed), to the sector $s_{T}^{z}=0$. We denote by $N_{f}(n)$ the minimal number of distinct (and nonzero) fundamental multispin-slip configurations that are retained at a given LSUB $n$ level of approximation after all such symmetries and conservation laws are taken into account. The number $N_{f}(n)$ typically still grows rapidly with $n$, and available computational resources then determine the maximum order $n$ that can be computed.

In the present case we are able to perform LSUB $n$ calculations up to the very high order $n=10$. Thus, for the spin- $\frac{1}{2}$ square-lattice bilayer model under consideration we have $N_{f}(10)=239021$ (443 813) when the CCM model state is chosen so that each monolayer has Néel (striped) AFM order. To derive and then to solve such larger sets of coupled nonlinear multinomial equations (11) for $S$ and linear equations (12) for $\tilde{S}$ we use both massive parallelization and large-scale supercomputer resources. In order to derive the equations (and see Ref. [82]) we also use a purpose-built and customized computer algebra package [112], without which it would not be possible to go to such large orders $n$ of LSUB $n$ truncation.

Since no approximations have been made in the evaluation of any finite-order LSUB $n$ truncation of our basic CCM equations, nor in the subsequent evaluation of any GS parameter of the system, our only approximation is now made at the last step where we extrapolate an LSUB $n$ sequence of approximants to the (exact) $n \rightarrow \infty$ limit. By now there is a great deal of empirical evidence on how to do so. For example, for the LSUB $n$ approximants $M(n)$ to the magnetic order parameter $M$ of Eq. (18), a well-tested scheme for systems with strong frustration, and/or for which the system has a QPT between states with and without magnetic LRO, has been found (see, e.g., Refs. [25-27,33,35,85,107,108,111] and references cited therein) to be given by

$$
M(n)=\mu_{0}+\mu_{1} n^{-1 / 2}+\mu_{2} n^{-3 / 2} .
$$

By fitting a sequence of LSUB $n$ approximants $M(n)$ to Eq. (19) we thus extract the corresponding extrapolated $\left(\mathrm{LSUB} \infty\right.$ ) value $\mu_{0}$ for $M$.

\section{RESULTS}

We show first in Fig. 2 our CCM results for the magnetic order parameter $M$ based on a model state in which each monolayer has Néel order. For values of the interlayer coupling parameter $\delta>0(\delta<0)$ the two layers are coupled so that NN spins are antiparallel (parallel). Results are shown as functions of $\delta$ in Figs. 2(a) and 2(b), respectively, for two fixed values of the intralayer frustration parameter $\kappa=0.3$ and 0.5 . These values are chosen to lie on either side of the critical value $\kappa_{c_{1}}(\delta=0) \approx 0.45$, at which Néel order melts in the monolayer. In both cases results are shown for even-order LSUB $n$ approximations with $4 \leqslant n \leqslant 10$, as well as for the $(\operatorname{LSUB} \infty)$ estimates $\mu_{0}$ obtained from fitting these data to the extrapolation scheme of Eq. (19). The LSUB2 data are omitted since, in principle, they are expected to be of too low order to fit well to such a three-term extrapolation scheme. Nevertheless, when separate fits are made that include them (i.e., to the LSUB $n$ data sets $n=\{2,4,6,8,10\}$ ), the extrapolated values for $\mu_{0}$ hardly change at all, thereby demonstrating the robustness of the fits. To enable readers to judge by eye for themselves how robust and accurate are our extrapolations, we also show in Fig. 2(a) the corresponding extrapolation (labeled LSUB $\infty^{\prime}$ ) based on the restricted LSUB $n$ data set $n=\{6,8,10\}$. Although a fit based on only three data points to a three-term extrapolation scheme such as that of Eq. (19) is not, a priori, expected to be as robust as one based on more data points, the agreement between the $\operatorname{LSUB} \infty$ and LSUB $\infty^{\prime}$ curves can be clearly seen. Similar agreement is observed for all other values of the intralayer frustration parameter $\kappa$.

Turning first to Fig. 2(a), the LSUB $\infty$ extrapolated curve exhibits all of the features we expect from our discussion in Sec. II. Thus, first, the cusp at $\delta=0$ (where $M \approx 0.17$ for the value $\kappa=0.3$ shown) is exactly as expected from the observation that as $|\delta|$ is increased from zero in either 

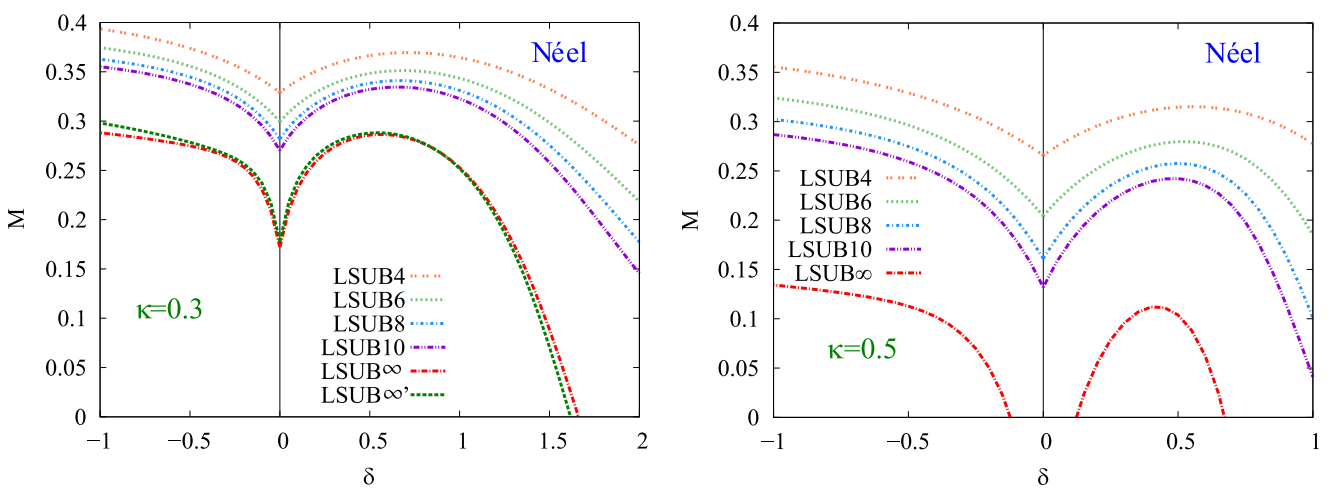

FIG. 2. CCM results for the GS magnetic order parameter $M$ vs the scaled interlayer exchange coupling constant $\delta \equiv J_{1}^{\perp} / J_{1}$ for the spin- $\frac{1}{2} J_{1}-J_{2}-J_{1}^{\perp}$ model on the square-lattice bilayer (with $J_{1}>0$ ), for two selected values of the intralayer frustration parameter $\kappa \equiv J_{2} / J_{1}$; (a) $\kappa=0.3$, and (b) $\kappa=0.5$. Results based on the Néel state on each monolayer, and the two layers coupled so that NN spins between them are antiparallel (parallel) to one another for $\delta>0(\delta<0)$, as CCM model state are shown in LSUB $n$ approximations with $n=4,6,8,10$, together with the corresponding LSUB $\infty$ extrapolated result based on Eq. (19) and the LSUB $n$ data sets $n=\{4,6,8,10\}$. For case (a) we also show the respective LSUB $\infty^{\prime}$ extrapolated curve using the restricted $\operatorname{LSUB} n$ data set $n=\{6,8,10\}$.

direction, the order is first enhanced due to the increase in the number of $\mathrm{NN}$ bonds and the consequent step toward increasing the dimensionality of the system. Second, we observe that as $\delta$ is increased further, in the regime $\delta>0$ of AFM coupling between the two layers, $M$ attains a maximum value of about 0.29 at a value $\delta \approx 0.57$ before the effects of interlayer dimerization become sufficiently strong to start to weaken the intralayer Néel order as $\delta$ is increased further beyond that point. This continues up to an upper critical value $\delta_{c_{1}}^{>}=\delta_{c_{1}}^{>}(\kappa)$, above which Néel order disappears entirely. For the value $\kappa=0.3$ shown in Fig. 2(a), this upper critical value is seen to be at $\delta_{c_{1}}^{>}(0.3) \approx 1.66$.

As the value of $\kappa$ is increased beyond 0.3 , the cusp at $\delta=0$ in the LSUB $\infty$ curve of Fig. 2(a) is lowered until, at the value $\kappa \approx 0.447$, it reaches the $\delta=0$ axis. This is precisely the value we obtain for $\kappa_{c_{1}}(\delta=0)$ within this same extrapolation scheme, using the $\operatorname{LSUB} n$ data set $n=\{4,6,8,10\}$. We note in passing that for the monolayer case $(\delta=0)$ it has also been possible to perform LSUB12 calculations [50]. The corresponding value obtained from fitting to the LSUB $n$ data set $n=\{4,6,8,10,12\}$ is $\kappa_{c_{1}}(\delta=0) \approx 0.454$ [50], which again demonstrates the accuracy and robustness of our extrapolations.

If we now slightly increase $\kappa$ beyond this value $\kappa_{c_{1}}(0)$, we obtain curves such as those shown in Fig. 2(b). Thus, for a certain range of values above $\kappa_{c_{1}}(0)$, for which Néel order is absent for the monolayer $(\delta=0)$, as $\delta$ is either decreased or increased from this value, Néel order becomes reestablished at certain critical values $\delta_{c_{1}}^{\mathrm{F}}(\kappa)$ for $\delta<0$ and $\delta_{c_{1}}^{<}(\kappa)$ for $\delta>0$. For the value $\kappa=0.5$ shown in Fig. 2(b), these values are seen to be $\delta_{c_{1}}^{\mathrm{F}}(0.5) \approx-0.12$ and $\delta_{c_{1}}^{<}(0.5) \approx 0.12$. Once again, as $\delta$ is now increased beyond the value $\delta_{c_{1}}^{<}(\kappa)$, Néel order is at first enhanced until $M$ attains a maximal value. For the value $\kappa=$ 0.5 shown in Fig. 2(b), this maximum value for $M$, for the case of AFM interlayer coupling, is about 0.11 at a value $\delta \approx 0.42$. Further increase in $\delta$ then reduces the magnetic order until it again melts entirely at a value $\delta_{c_{1}}^{>}(\kappa)$. For $\kappa=0.5$ this upper critical value is seen from Fig. $2(\mathrm{~b})$ to be at $\delta_{c_{1}}^{>}(0.5) \approx 0.67$.

If we continue to increase $\kappa$ slowly beyond the value $\kappa=$ 0.5 shown in Fig. 2(b), the lower and upper critical values $\delta_{c_{1}}^{<}(\kappa)$ and $\delta_{c_{1}}^{>}(\kappa)$ move toward one another until at some value $\kappa_{1}^{\max } \approx 0.535$ they merge, $\delta_{c_{1}}^{<}\left(\kappa_{1}^{\max }\right)=\delta_{c_{1}}^{>}\left(\kappa_{1}^{\max }\right) \approx 0.3$. Néel order is then wholly absent for any value $\kappa>\kappa_{1}^{\max }$ of the intralayer frustration parameter and for any value $\delta>0$ of the AFM interlayer coupling.

Corresponding results to those shown in Fig. 2, which are based on a CCM model state with Néel order on each monolayer, as in Fig. 1(b), are now shown in Fig. 3 based on a corresponding model state with striped AFM order on each monolayer, as in Fig. 1(c). The two layers are again coupled so that $\mathrm{NN}$ interlayer spins are antiparallel for $\delta>0$ and parallel for $\delta<0$. The two values of $\kappa$ shown, viz., $\kappa=0.7$ in Fig. 3(a) and $\kappa=0.57$ in Fig. 3(b) are now chosen to lie on either side of the critical value $\kappa_{c_{2}}(\delta=0) \approx 0.59$ for which striped order melts in the monolayer. In Fig. 3(a) we also show the corresponding LSUB $\infty^{\prime}$ extrapolated curve based on the restricted LSUB $n$ data set $n=\{6,8,10\}$. The agreement with the LSUB $\infty$ curve based on the full LSUB $n$ data set $n=\{4,6,8,10\}$ is again observed to be excellent. Similar levels of agreement are found for all other values of $\kappa$. Once again, for the special case of the monolayer LSUB12 calculations have also been performed [50] based on the striped model state. In this case, we find $\kappa_{c_{2}}(0) \approx$ 0.587 based on the extrapolation of Eq. (19) with the LSUBn data set $n=\{4,6,8,10\}$ as input, while the corresponding result based on the set $n=\{4,6,8,10,12\}$ yielded the almost identical value $\kappa_{c_{2}}(0) \approx 0.588$ [50].

The similarity between Figs. 2(a) and 3(a), and also between Figs. 2(b) and 3(b), is self-evident. In this case, we find that the lower and upper critical values $\delta_{c_{2}}^{<}(\kappa)$ and $\delta_{c_{2}}^{>}(\kappa)$, as seen in Fig. 3(b) in the region $\delta>0$ for the value $\kappa=$ $0.57<\kappa_{c_{2}}(0)$ again move toward one another as $\kappa$ is now slowly decreased beyond this value, until they merge at a value $\kappa_{2}^{\min } \approx 0.555$, where $\delta_{c_{2}}^{<}\left(\kappa_{2}^{\min }\right)=\delta_{c_{2}}^{>}\left(\kappa_{2}^{\min }\right) \approx 0.3$. For all values $\kappa<\kappa_{2}^{\text {min }}$ of the intralayer frustration parameter, striped order is then absent, whatever the value $\delta>0$ of the AFM interlayer coupling.

In Figs. 4(a) and 4(b) we now show sets of extrapolated $(\mathrm{LSUB} \infty)$ curves for the magnetic order parameter $M$ as a 

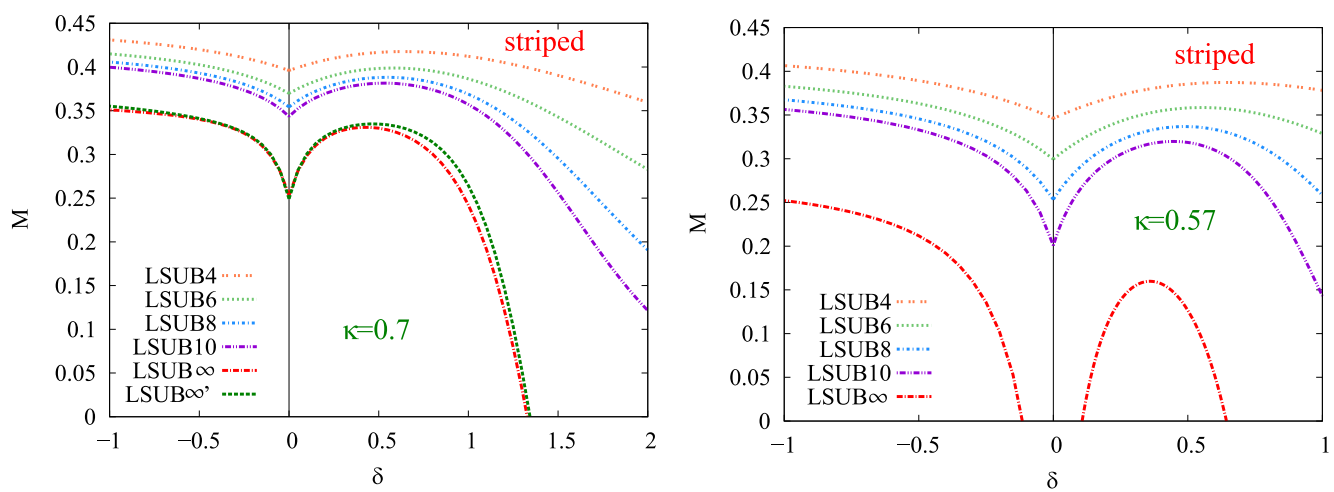

FIG. 3. CCM results for the GS magnetic order parameter $M$ vs the scaled interlayer exchange coupling constant $\delta \equiv J_{1}^{\perp} / J_{1}$ for the spin- $\frac{1}{2} J_{1}-J_{2}-J_{1}^{\perp}$ model on the square-lattice bilayer (with $J_{1}>0$ ), for two selected values of the intralayer frustration parameter $\kappa \equiv J_{2} / J_{1}$; (a) $\kappa=0.7$, and (b) $\kappa=0.57$. Results based on the striped state on each monolayer, and the two layers coupled so that NN spins between them are antiparallel (parallel) to one another for $\delta>0(\delta<0)$, as CCM model state are shown in LSUB $n$ approximations with $n=4,6,8,10$, together with the corresponding LSUB $\infty$ extrapolated result based on Eq. (19) and the LSUB $n$ data sets $n=\{4,6,8,10\}$. For case (a) we also show the respective LSUB $\infty^{\prime}$ extrapolated curve using the restricted LSUB $n$ data set $n=\{6,8,10\}$.

function of the interlayer coupling parameter $\delta$ for each layer with Néel order and striped order, respectively, for various fixed values of the intralayer frustration $\kappa$. These correspond, respectively, to curves such as those shown in Figs. 2 and 3. From Fig. 4(b) it is clear that the position of the cusp at $\delta=0$ for the striped-ordered phase rather rapidly approaches a limiting value for $M$ as $\kappa$ is increased. This corresponds to the limit $(\kappa \rightarrow \infty)$ of the model where each layer corresponds to two independent, and equivalent, interpenetrating square sublattices, each of which is Néel ordered. A recent CCM calculation [113] for the spin- $\frac{1}{2}$ Heisenberg antiferromagnet on the square lattice utilized an LSUB $n$ data set $n=\{4,6,8,10,12\}$ to yield the extrapolated value $M \approx 0.3093$. By comparison, we find here the remarkably close value $M(\delta=0) \approx 0.3094$ from using Eq. (19) with the LSUB $n$ data set $n=\{4,6,8,10\}$, and as shown in Fig. 4(b), for the curve $\kappa=1.0$, which value is itself already extremely close to the limiting value obtained as $\kappa \rightarrow \infty$.

We also note from Fig. 4(a) that for the case of zero intralayer frustration $(\kappa=0)$, our extrapolation of Eq. (19) leads to an upper critical value $\delta_{c_{1}}^{>}(\kappa=0) \approx 2.84$ of the interlayer coupling parameter, beyond which Néel order melts and a phase with IDVBC order is stabilized. This may be compared with the corresponding value of 2.5220 obtained from a large-scale QMC simulation [96] of the spin- $\frac{1}{2} J_{1}-J_{1}^{\perp}$ model discussed in Sec. II. In this context, it is worth noting that the location of the phase boundary is less accurately determined in our CCM calculations for the region where the order-disorder transition is essentially driven by singlet dimerization than in the region where it is essentially due to frustration.

Particularly in the half-plane $\delta<0$, corresponding to FM interlayer coupling, it is also convenient to investigate the magnetic order parameter $M$ for the two quasiclassical phases with AFM orderings on each monolayer as functions of the intralayer frustration parameter $\kappa$, for various fixed values of the interlayer coupling parameter $\delta$. In Fig. 5 we show a set of such curves for both AFM monolayer orderings. The curves for $\delta=0$ again exhibit the corresponding critical points $\kappa_{c_{1}}(\delta=0) \approx 0.447$ and $\kappa_{c_{2}}(\delta=0) \approx 0.587$ for the melting of Néel and striped order, respectively, for the spin$\frac{1}{2} J_{1}-J_{2}$ model on the square-lattice monolayer. We also show
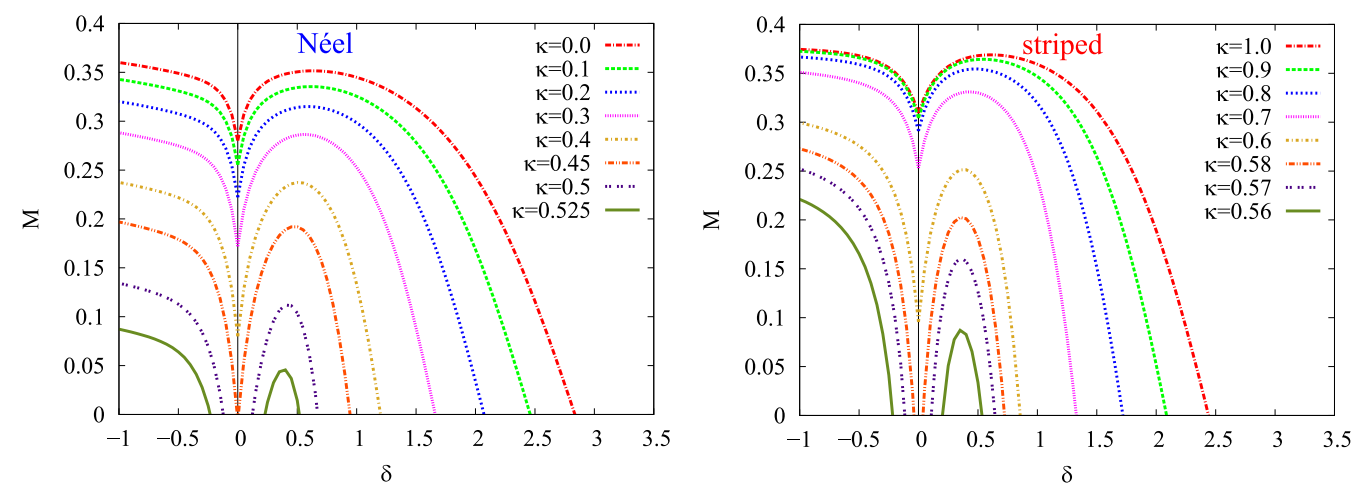

FIG. 4. CCM results for the GS magnetic order parameter $M$ vs the scaled interlayer exchange coupling constant $\delta \equiv J_{1}^{\perp} / J_{1}$ for the spin- $\frac{1}{2} J_{1}-J_{2}-J_{1}^{\perp}$ model on the square-lattice bilayer (with $J_{1}>0$ ), for a variety of values of the intralayer frustration parameter $\kappa \equiv J_{2} / J_{1}$, using (a) the Néel state and (b) the striped state as the CCM model state on each monolayer, and the two layers coupled so that NN spins between them are antiparallel (parallel) to one another for $\delta>0(\delta<0)$. In each case we show extrapolated results, obtained from using Eq. (19) with the respective LSUB $n$ data sets $n=\{4,6,8,10\}$. 


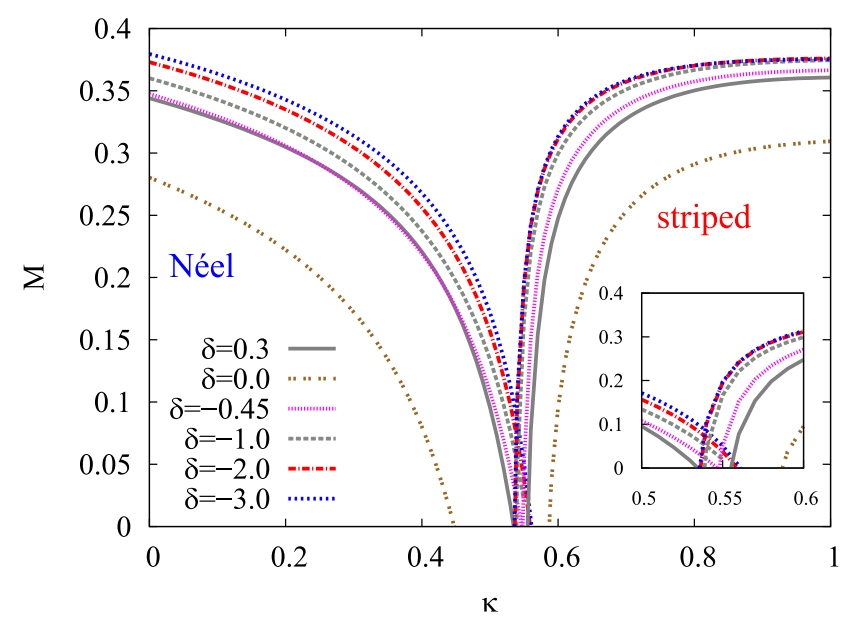

FIG. 5. CCM results for the GS magnetic order parameter $M$ vs the intralayer frustration parameter $\kappa \equiv J_{2} / J_{1}$ for the spin- $\frac{1}{2} J_{1}-J_{2}-J_{1}^{\perp}$ model on the square-lattice bilayer (with $J_{1}>0$ ), for a variety of values of the scaled interlayer exchange coupling constant $\delta \equiv J_{1}^{\perp} / J_{1}$, using both the Néel state and the striped state independently as the CCM model state on each monolayer, and the two layers coupled so that NN spins between them are antiparallel to one another in the cases where $\delta>0$ and parallel to one another in the cases where $\delta<0$. In each case we show extrapolated results, obtained from using Eq. (19) with the respective LSUB $n$ data sets $n=\{4,6,8,10\}$.

the corresponding curves for $\delta=0.3$ in the region of AFM coupling between the layers, which is approximately the value of $\delta$ for which the phase boundaries of the two quasiclassical phases are closest together in this region $\delta>0$. Thus, at $\delta=0.3$, the paramagnetic state exists only in the very narrow regime $0.535 \lesssim \kappa \lesssim 0.555$ of the frustration parameter.

We also show in Fig. 5 similar curves for various values of $\delta$ in the half-plane $\delta<0$, which corresponds to FM interlayer coupling. One sees clearly that for all values $\delta<\delta_{\mathrm{T}}^{\mathrm{F}} \approx-0.45$, the respective curves $M=M(\kappa)$ for the two quasiclassical phases cross one another at a value $\kappa^{\mathrm{F}}(\delta)>0$, indicating a direct first-order transition between them. The value $\kappa^{\mathrm{F}}(\delta)$ is seen to be almost independent of $\delta$ in this regime. For example, $\kappa^{\mathrm{F}}(-3) \approx 0.539$, which may be compared with the expected value, $\lim _{\delta \rightarrow-\infty} \kappa^{\mathrm{F}}(\delta) \approx 0.549$, viz., the value that corresponds to the critical coupling for the direct transition between the two states in the spin- $1 J_{1}-J_{2}$ model on the squarelattice monolayer [109], as discussed in Sec. II. From Fig. 5 we see that the QTP where the Néel, striped, and paramagnetic phases meet is situated at $\left(\kappa_{\mathrm{T}}^{\mathrm{F}}, \delta_{\mathrm{T}}^{\mathrm{F}}\right) \approx(0.547,-0.45)$.

Of course, in view of the observation that the direct transition between the two quasiclassical phases for $\delta \lesssim-0.45$ is of first-order type, we can also corroborate our results in this regime by using the fact that the GS energies of the two states should also cross at the phase boundary. We use the appropriate extrapolation scheme for the $\mathrm{LSUB} n$ approximants $e(n)$ to the GS energy per spin $e \equiv E / N$, which is well known to be given by

$$
e(n)=e_{0}+e_{1} n^{-2}+e_{2} n^{-4} .
$$

We may thus make use of Eq. (20) with the same LSUB $n$ input data sets $n=\{4,6,8,10\}$ as used in Fig. 5 to corroborate the

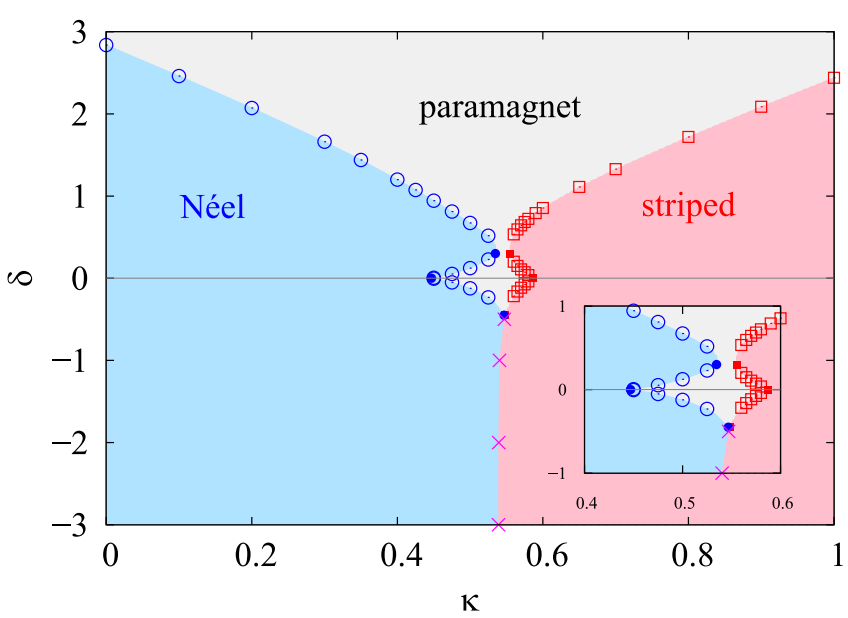

FIG. 6. $T=0$ phase diagram of the spin- $\frac{1}{2} J_{1}-J_{2}-J_{1}^{\perp}$ model on the bilayer square lattice with $J_{1}>0, \delta \equiv J_{1}^{\perp} / J_{1}$, and $\kappa \equiv J_{2} / J_{1}$. The blue and pink regions are the quasiclassical phases with AFM Néel and striped orders, respectively, while in the gray region quasiclassical collinear order is absent. The filled and empty circle (and square) symbols are points at which the extrapolated GS magnetic order parameter $M$ for the Néel (and striped) phases vanishes, for specified values of $\delta$ and $\kappa$, respectively. By contrast, the cross $(\times)$ symbols indicate points at which the corresponding two curves $M=M(\kappa)$, for a specified value of $\delta$, cross one another. In each case, the Néel or striped state on each monolayer is used as CCM model state, and Eq. (19) is used for the extrapolations with the corresponding LSUB $n$ data sets $n=\{4,6,8,10\}$.

points shown in Fig. 5 by cross $(\times)$ symbols, which denote the points where the respective $M=M(\kappa)$ curves for the two quasiclassical phases cross one another for a given value of $\delta$ at the value $\kappa=\kappa^{\mathrm{F}}(\delta)$. We find, for example, at the value $\delta=-3$, the two corresponding LSUB $\infty$ extrapolated curves $e=e(\kappa)$ cross one another at the value $\kappa \approx 0.558$, which may be compared with the value $\kappa^{\mathrm{F}}(-3) \approx 0.539$ cited above, where the respective $M=M(\kappa)$ curves cross. The agreement between these two essentially independent calculations is good. This clearly also provides internal confirmation of the robustness of the extrapolation schemes that form the sole approximation made in our results.

By combining results from curves such as those shown in Figs. 2-5 to extract the points where the extrapolated $(\mathrm{LSUB} \infty)$ GS magnetic order parameter $M$ vanishes for the two quasiclassical AFM phases, we may finally construct the zero-temperature $(T=0)$ quantum phase diagram of our spin $-\frac{1}{2} J_{1}-J_{2}-J_{1}^{\perp}$ model on the bilayer square lattice. It is shown in Fig. 6 in the $\kappa-\delta$ half-plane with $\kappa>0$. We note that different symbols are used in Fig. 6 to distinguish between points on the phase boundaries that have been extracted from calculations done at fixed values of $\kappa$ and those extracted from calculations done at fixed values of $\delta$. It is clear by visual inspection that these two sets of critical points lie very accurately on a smooth boundary curve for each collinear AFM state. This again provides good internal evidence that our extrapolations are robust and accurate.

Our results are now discussed and summarized in Sec. V. 


\section{DISCUSSION AND SUMMARY}

We see from Fig. 6 that the phase boundary for each of the AFM quasiclassical states is rather accurately linear in the $\kappa-\delta$ plane for large enough values of AFM interlayer coupling (viz., for $\delta \gtrsim 1.5$ ). For the Néel phase boundary the slope of the curve at large values of $\delta$ is $d \delta / d \kappa \approx-3.9$, while the corresponding value for the striped phase boundary is $d \delta / d \kappa \approx 3.7$. If this linear behavior would continue unchanged to smaller values of $\delta$, the two curves would cross at a point $(\kappa, \delta) \approx(0.54,0.7)$. Instead, as we see from Fig. 6 , the two curves turn against each other before this point, in a typical "avoided crossing" manner, although they do approach one another rather closely in the region around $\delta \approx 0.3$. This has the effect that the entire disordered paramagnetic regime in Fig. 6 is singly connected.

At small values of $\delta$, both phase boundaries exhibit the reentrant behavior expected from our discussion in Sec. II, with both displaying cusps at $\delta=0$. However, a closer inspection of Fig. 6 shows that the nature of the cusps is quite different for the two phase boundaries, with that on the Néel side much "sharper" than its counterpart on the striped side. More quantitatively, on the striped side the slopes of the curves on each side of the cusp at the point $\kappa=$ $\kappa_{c_{2}}(0), \delta=0$ are clearly nonzero. By contrast, on the Néel side the corresponding slopes of the curves of the cusp at the point $\kappa=\kappa_{c_{1}}(0), \delta=0$ appear to be zero (or very close) to zero. This difference would certainly explain why the critical parameter $\kappa_{c_{1}}(0)$ is more difficult to calculate accurately than the corresponding parameter $\kappa_{c_{2}}(0)$ for the spin- $\frac{1}{2} J_{1}-J_{2}$ model on the square lattice, as has been discussed previously in Sec. II. The difference is surely also a direct reflection of the different natures of the two QPTs in that model. Thus, our results are in clear accord with the consensual view that, while the transition at $\kappa_{c_{2}}(0)$ between the striped and paramagnetic phases is a first-order one, that at $\kappa_{c_{1}}(0)$ between the Néel and paramagnetic phases is continuous.

In the half-plane $\delta<0$ the phase boundaries of the two quasiclassical AFM phases end at a QTP where they meet a line of direct first-order transitions between the two phases. Starting at the QTP, which we have calculated as being located at $\left(\kappa_{\mathrm{T}}^{\mathrm{F}} \approx 0.547, \delta_{\mathrm{T}}^{\mathrm{F}} \approx-0.45\right)$, this line of first-order transitions is very nearly a vertical straight line in the $\kappa-\delta$ plane. At large negative values of $\delta$ it approaches the value $\kappa^{\mathrm{F}}(\delta \rightarrow$ $-\infty) \approx 0.539$, which is itself an accurate estimate of the QCP for the direct transition between the two quasiclassical phases of the spin-1 $J_{1}-J_{2}$ model on the square lattice.

It is completely beyond the scope of this paper to investigate in detail the nature of the phases in the (gray shaded) paramagnetic regime in Fig. 6, outside the respective regimes in which we have calculated that the Néel state or the striped state on each monolayer form the stable GS phase. Nevertheless, we conclude with a few comments on this issue.

We have already discussed in Sec. I the lack of overall consensus for the nature of the GS phase or phases in the region $\kappa_{c_{1}}(0)<\kappa<\kappa_{c_{2}}(0)$ for the spin- $\frac{1}{2} J_{1}-J_{2}$ model on the square-lattice monolayer. However, the results of two independent high-order techniques, viz., the CCM [27,50] and the DMRG method [42], applied to the model are both compatible with the existence of two phases in this paramagnetic region. Both CCM and DMRG calculations also agree on the critical values $\kappa_{c_{1}}(0) \approx 0.45(1)$ and $\kappa_{c_{2}}(0) \approx 0.60(1)$. Furthermore, both can be consistently interpreted with the hypothesis of a gapped plaquette-ordered VBC (PVBC) ground state in the region $0.5 \lesssim \kappa<\kappa_{c_{2}}(0)$, and a ground state in the region $\kappa_{c_{1}}(0)<\kappa \lesssim 0.5$ that could be a gapless QSL state. In view of the single connectedness of the entire paramagnetic regime that we have found, it is clear that, based on the above scenario being true for $\delta=0$, this paramagnetic regime for the bilayer should include at least three phases, viz., QSL, PVBC, and IDVBC. It will clearly be of great future interest to study the boundaries of these phases in detail.

\section{ACKNOWLEDGMENT}

We thank the University of Minnesota Supercomputing Institute for the grant of supercomputing facilities, on which some of the work reported here was performed.
[1] P. Chandra and B. Doucot, Possible spin-liquid state at large $S$ for the frustrated square Heisenberg lattice, Phys. Rev. B 38, 9335(R) (1988).

[2] E. Dagotto and A. Moreo, Phase Diagram of the Frustrated Spin- $\frac{1}{2}$ Heisenberg Antiferromagnet in Two Dimensions, Phys. Rev. Lett. 63, 2148 (1989).

[3] M. P. Gelfand, R. R. P. Singh, and D. A. Huse, Zerotemperature ordering in two-dimensional frustrated quantum Heisenberg antiferromagnets, Phys. Rev. B 40, 10801 (1989).

[4] S. Sachdev and R. N. Bhatt, Bond-operator representation of quantum spins: Mean-field theory of frustrated quantum Heisenberg antiferromagnets, Phys. Rev. B 41, 9323 (1990).

[5] A. V. Chubukov and Th. Jolicoeur, Dimer stability region in a frustrated quantum Heisenberg antiferromagnet, Phys. Rev. B 44, 12050(R) (1991).

[6] N. Read and S. Sachdev, Large- $N$ Expansion for Frustrated Quantum Antiferromagnets, Phys. Rev. Lett. 66, 1773 (1991).
[7] J. Richter, Zero-temperature magnetic ordering in the inhomogeneously frustrated quantum Heisenberg antiferromagnet on a square lattice, Phys. Rev. B 47, 5794 (1993).

[8] J. Richter, N. B. Ivanov, and K. Retzlaff, On the violation of Marshall-Peierls sign rule in the frustrated $J_{1}-J_{2}$ Heisenberg antiferromagnet, Europhys. Lett. 25, 545 (1994).

[9] N. B. Ivanov and J. Richter, $J_{1}-J_{2}$ quantum Heisenberg antiferromagnet: improved spin-wave theories versus exactdiagonalization data, J. Phys.: Condens. Matter 6, 3785 (1994).

[10] H. J. Schulz, T. A. L. Ziman, and D. Poilblanc, Magnetic order and disorder in the frustrated quantum Heisenberg antiferromagnet in two dimensions, J. Phys. I (France) 6, 675 (1996)

[11] J. Oitmaa and Z. Weihong, Series expansion for the $J_{1}-J_{2}$ Heisenberg antiferromagnet on a square lattice, Phys. Rev. B 54, 3022 (1996). 
[12] M. E. Zhitomirsky and K. Ueda, Valence-bond crystal phase of a frustrated spin- $\frac{1}{2}$ square-lattice antiferromagnet, Phys. Rev. B 54, 9007 (1996).

[13] A. E. Trumper, L. O. Manuel, C. J. Gazza, and H. A. Ceccatto, Schwinger-Boson Approach to Quantum Spin Systems: Gaussian Fluctuations in the "natural" Gauge, Phys. Rev. Lett. 78, 2216 (1997).

[14] R. F. Bishop, D. J. J. Farnell, and J. B. Parkinson, Phase transitions in the spin-half $J_{1}-J_{2}$ model, Phys. Rev. B 58, 6394 (1998).

[15] R. R. P. Singh, Zheng Weihong, C. J. Hamer, and J. Oitmaa, Dimer order with striped correlations in the $J_{1}-J_{2}$ Heisenberg model, Phys. Rev. B 60, 7278 (1999).

[16] V. N. Kotov, J. Oitmaa, O. P. Sushkov, and Z. Weihong, Lowenergy singlet and triplet excitations in the spin-liquid phase of the two-dimensional $J_{1}-J_{2}$ model, Phys. Rev. B 60, 14613 (1999).

[17] L. Capriotti and S. Sorella, Spontaneous Plaquette Dimerization in the $J_{1}-J_{2}$ Heisenberg Model, Phys. Rev. Lett. 84, 3173 (2000).

[18] L. Capriotti, F. Becca, A. Parola, and S. Sorella, Resonating Valence Bond Wave Functions for Strongly Frustrated Spin Systems, Phys. Rev. Lett. 87, 097201 (2001).

[19] K. Takano, Y. Kito, Y. Ōno, and K. Sano, Nonlinear $\sigma$ Model Method for the $J_{1}-J_{2}$ Heisenberg Model: Disordered Ground State with Plaquette Symmetry, Phys. Rev. Lett. 91, 197202 (2003).

[20] T. Roscilde, A. Feiguin, A. L. Chernyshev, S. Liu, and S. Haas, Anisotropy-Induced Ordering in the Quantum $J_{1}-J_{2}$ Antiferromagnet, Phys. Rev. Lett. 93, 017203 (2004).

[21] V. Lante and A. Parola, Ising phase in the $J_{1}-J_{2}$ Heisenberg model, Phys. Rev. B 73, 094427 (2006).

[22] J. Sirker, Z. Weihong, O. P. Sushkov, and J. Oitmaa, $J_{1}-J_{2}$ model: First-order phase transition versus deconfinement of spinons, Phys. Rev. B 73, 184420 (2006).

[23] D. Schmalfuß, R. Darradi, J. Richter, J. Schulenburg, and D. Ihle, Quantum $J_{1}-J_{2}$ Antiferromagnet on a Stacked Square Lattice: Influence of the Interlayer Coupling on the GroundState Magnetic Ordering, Phys. Rev. Lett. 97, 157201 (2006).

[24] M. Mambrini, A. Läuchli, D. Poilblanc, and F. Mila, Plaquette valence-bond crystal in the frustrated Heisenberg quantum antiferromagnet on the square lattice, Phys. Rev. B 74, 144422 (2006).

[25] R. F. Bishop, P. H. Y. Li, R. Darradi, and J. Richter, The quantum $J_{1}-J_{1}^{\prime}-J_{2}$ spin-1/2 Heisenberg model: influence of the interchain coupling on the ground-state magnetic ordering in two dimensions, J. Phys.: Condens. Matter 20, 255251 (2008).

[26] R. F. Bishop, P. H. Y. Li, R. Darradi, J. Schulenburg, and J. Richter, Effect of anisotropy on the ground-state magnetic ordering of the spin-half quantum $J_{1}^{X X Z}-J_{2}^{X X Z}$ model on the square lattice, Phys. Rev. B 78, 054412 (2008).

[27] R. Darradi, O. Derzhko, R. Zinke, J. Schulenburg, S. E. Krüger, and J. Richter, Ground state phases of the spin-1/2 $J_{1}-J_{2}$ Heisenberg antiferromagnet on the square lattice: A high-order coupled cluster treatment, Phys. Rev. B 78, 214415 (2008).

[28] L. Isaev, G. Ortiz, and J. Dukelsky, Hierarchical mean-field approach to the $J_{1}-J_{2}$ Heisenberg model on a square lattice, Phys. Rev. B 79, 024409 (2009).
[29] V. Murg, F. Verstraete, and J. I. Cirac, Exploring frustrated spin systems using projected entangled pair states, Phys. Rev. B 79, 195119 (2009).

[30] A. Ralko, M. Mambrini, and D. Poilblanc, Generalized quantum dimer model applied to the frustrated Heisenberg model on the square lattice: Emergence of a mixed columnarplaquette phase, Phys. Rev. B 80, 184427 (2009).

[31] J. Richter and J. Schulenburg, The spin-1/2 $J_{1}-J_{2}$ Heisenberg antiferromagnet on the square lattice: Exact diagonalization for $N=40$ spins, Eur. Phys. J. B 73, 117 (2010).

[32] J. Reuther and P. Wölfle, $J_{1}-J_{2}$ frustrated two-dimensional Heisenberg model: Random phase approximation and functional renormalization group, Phys. Rev. B 81, 144410 (2010).

[33] J. Reuther, P. Wölfle, R. Darradi, W. Brenig, M. Arlego, and J. Richter, Quantum phases of the planar antiferromagnetic $J_{1}-J_{2}-J_{3}$ Heisenberg model, Phys. Rev. B 83, 064416 (2011).

[34] Ji-Feng Yu and Ying-Jer Kao, Spin- $\frac{1}{2} J_{1}-J_{2}$ Heisenberg antiferromagnet on a square lattice: A plaquette renormalized tensor network study, Phys. Rev. B 85, 094407 (2012).

[35] O. Götze, S. E. Krüger, F. Fleck, J. Schulenburg, and J. Richter, Ground-state phase diagram of the spin- $\frac{1}{2}$ squarelattice $J_{1}-J_{2}$ model with plaquette structure, Phys. Rev. B 85 224424 (2012).

[36] Hong-Chen Jiang, Hong Yao, and Leon Balents, Spin liquid ground state of the spin- $\frac{1}{2}$ square $J_{1}-J_{2}$ Heisenberg model, Phys. Rev. B 86, 024424 (2012).

[37] F. Mezzacapo, Ground-state phase diagram of the quantum $J_{1}-J_{2}$ model on the square lattice, Phys. Rev. B 86, 045115 (2012).

[38] T. Li, F. Becca, W. Hu, and S. Sorella, Gapped spin-liquid phase in the $J_{1}-J_{2}$ Heisenberg model by a bosonic resonating valence-bond ansatz, Phys. Rev. B 86, 075111 (2012).

[39] L. Wang, D. Poilblanc, Z.-C. Gu, X.-G. Wen, and F. Verstraete, Constructing a Gapless Spin-Liquid State for the Spin$1 / 2 J_{1}-J_{2}$ Heisenberg Model on a Square Lattice, Phys. Rev. Lett. 111, 037202 (2013).

[40] X. Zhang and K. S. D. Beach, Resonating valence bond trial wave functions with both static and dynamically determined Marshall sign structure, Phys. Rev. B 87, 094420 (2013).

[41] W.-J. Hu, F. Becca, A. Parola, and S. Sorella, Direct evidence for a gapless $Z_{2}$ spin liquid by frustrating Néel antiferromagnetism, Phys. Rev. B 88, 060402(R) (2013).

[42] S.-S. Gong, W. Zhu, D. N. Sheng, O. I. Motrunich, and M. P. A. Fisher, Plaquette Ordered Phase and Quantum Phase Diagram in the Spin- $\frac{1}{2} J_{1}-J_{2}$ Square Heisenberg Model, Phys. Rev. Lett. 113, 027201 (2014).

[43] R. L. Doretto, Plaquette valence-bond solid in the squarelattice $J_{1}-J_{2}$ antiferromagnet Heisenberg model: A bond operator approach, Phys. Rev. B 89, 104415 (2014).

[44] Y. Qi and Z.-C. Gu, Continuous phase transition from Néel state to $Z_{2}$ spin-liquid state on a square lattice, Phys. Rev. B 89, 235122 (2014).

[45] A. Metavitsiadis, D. Sellmann, and S. Eggert, Spin-liquid versus dimer phases in an anisotropic $J_{1}-J_{2}$ frustrated square antiferromagnet, Phys. Rev. B 89, 241104(R) (2014).

[46] Y.-Z. Ren, N.-H. Tong, and X.-C. Xie, Cluster mean-field theory study of $J_{1}-J_{2}$ Heisenberg model on a square lattice, J. Phys.: Condens. Matter 26, 115601 (2014). 
[47] L. Wang, Correlated valence bond state and its study of the spin-1/2 $J_{1}-J_{2}$ antiferromagnetic Heisenberg model on a square lattice, arXiv:1402.3564.

[48] C.-P. Chou and H.-Y. Chen, Simulating a two-dimensional frustrated spin system with fermionic resonating-valence-bond states, Phys. Rev. B 90, 041106(R) (2014).

[49] S. Morita, R. Kaneko, and M. Imada, Quantum spin liquid in spin-1/2 $J_{1}-J_{2}$ Heisenberg model on square lattice: Many-variable variational Monte Carlo study combined with quantum-number projections, J. Phys. Soc. Jpn. 84, 024720 (2015).

[50] J. Richter, R. Zinke, and D. J. J. Farnell, The spin-1/2 squarelattice $J_{1}-J_{2}$ model: the spin-gap issue, Eur. Phys. J. B 88, 2 (2015).

[51] L. Wang, Z.-C. Gu, F. Verstraete, and X.-G. Wen, Tensorproduct state approach to spin- $\frac{1}{2}$ square $J_{1}-J_{2}$ antiferromagnetic Heisenberg model: Evidence for deconfined quantum criticality, Phys. Rev. B 94, 075143 (2016).

[52] D. Poilblanc and M. Mambrini, Quantum critical phase with infinite projected entangled paired states, Phys. Rev. B 96, 014414 (2017).

[53] R. Haghshenas and D. N. Sheng, U(1)-symmetric infinite projected entangled-pair states study of the spin- $1 / 2$ square $J_{1}-J_{2}$ Heisenberg model, Phys. Rev. B 97, 174408 (2018).

[54] S.-L. Yu, W. Wang, Z.-Y. Dong, Z.-J. Yao, and J.-X. Li, Deconfinement of spinons in frustrated spin systems: Spectral perspective, Phys. Rev. B 98, 134410 (2018).

[55] L. Wang and A. W. Sandvik, Critical Level Crossings and Gapless Spin Liquid in the Square-Lattice Spin-1/2 $J_{1}-J_{2}$ Heisenberg Antiferromagnet, Phys. Rev. Lett. 121, 107202 (2018).

[56] W.-Y. Liu, S. Dong, C. Wang, Y. Han, H. An, G.-C. Guo, and L. He, Gapless spin liquid ground state of the spin$\frac{1}{2} J_{1}-J_{2}$ Heisenberg model on square lattices, Phys. Rev. B 98, 241109(R) (2018).

[57] P. W. Anderson, The resonating valence bond state in $\mathrm{La}_{2} \mathrm{CuO}_{4}$ and superconductivity, Science 235, 1196 (1987).

[58] P. A. Lee, N. Nagaosa, and X.-G. Wen, Doping a Mott insulator: Physics of high-temperature superconductivity, Rev. Mod. Phys. 78, 17 (2006).

[59] R. Melzi, P. Carretta, A. Lascialfari, M. Mambrini, M. Troyer, P. Millet, and F. Mila, $\mathrm{Li}_{2} \mathrm{VO}(\mathrm{Si}, \mathrm{Ge}) \mathrm{O}_{4}$, a Prototype of a TwoDimensional Frustrated Quantum Heisenberg Antiferromagnet, Phys. Rev. Lett. 85, 1318 (2000); R. Melzi, S. Aldrovandi, F. Tedoldi, P. Carretta, P. Millet, and F. Mila, Magnetic and thermodynamic properties of $\mathrm{Li}_{2} \mathrm{VOSiO}_{4}$ : A two-dimensional $S=1 / 2$ frustrated antiferromagnet on a square lattice, Phys. Rev. B 64, 024409 (2001); H. Rosner, R. R. P. Singh, W. H. Zheng, J. Oitmaa, and W. E. Pickett, High-temperature expansions for the $J_{1}-J_{2}$ Heisenberg models: Applications to ab initio calculated models for $\mathrm{Li}_{2} \mathrm{VOSiO}_{4}$ and $\mathrm{Li}_{2} \mathrm{VOGeO}_{4}$, ibid. 67 , 014416 (2003).

[60] Y. Todate, W. Higemoto, K. Nishiyama, and K. Hirota, Magnetic ordering in ordered complex $\mathrm{Cu}$ perovskite probed by $\mu$ SR and neutron diffraction, J. Phys. Chem. Solids 68, 2107 (2007).

[61] S. Vasala, H. Saadaoui, E. Morenzoni, O. Chmaissem, T.-S. Chan, J.-M. Chen, Y.-Y. Hsu, H. Yamauchi, and M. Karppinen, Characterization of magnetic properties of $\mathrm{Sr}_{2} \mathrm{CuWO}_{6}$ and $\mathrm{Sr}_{2} \mathrm{CuMoO}_{6}$, Phys. Rev. B 89, 134419 (2014).
[62] S. Vasala, M. Avdeev, S. Danilkin, O. Chmaissem, and M. Karppinen, Magnetic structure of $\mathrm{Sr}_{2} \mathrm{CuWO}_{6}$, J. Phys.: Condens. Matter 26, 496001 (2014).

[63] T. Koga, N. Kurita, M. Avdeev, S. Danilkin, T. J. Sato, and H. Tanaka, Magnetic structure of the $S=\frac{1}{2}$ quasi-twodimensional square-lattice Heisenberg antiferromagnet $\mathrm{Sr}_{2}$ $\mathrm{CuTeO}_{6}$, Phys. Rev. B 93, 054426 (2016).

[64] P. Chandra, P. Coleman, and A. I. Larkin, Ising Transition in Frustrated Heisenberg Models, Phys. Rev. Lett. 64, 88 (1990).

[65] J. Villain, A magnetic analogue of stereoisomerism: application to helimagnetism in two dimensions, J. Phys. (France) 38, 385 (1977); J. Villain, R. Bidaux, J.-P. Carton, and R. Conte, Order as an effect of disorder, ibid. 41, 1263 (1980).

[66] E. F. Shender, Antiferromagnetic garnets with fluctuationally interacting sublattices, Zh. Eksp. Teor. Fiz. 83, 326 (1982) [Sov. Phys.-JETP 56, 178 (1982)].

[67] A. W. Sandvik, Finite-size scaling and boundary effects in two-dimensional valence-bond solids, Phys. Rev. B 85, 134407 (2012).

[68] F. Coester, Bound states of a many-particle system, Nucl. Phys. 7, 421 (1958).

[69] F. Coester and H. Kümmel, Short-range correlations in nuclear wave functions, Nucl. Phys. 17, 477 (1960).

[70] Jiři Čižek, On the correlation problem in atomic and molecular systems. Calculation of wavefunction components in Urselltype expansion using quantum-field theoretical methods, J. Chem. Phys. 45, 4256 (1966).

[71] H. Kümmel, K. H. Lührmann, and J. G. Zabolitzky, Manyfermion theory in $\exp S$ (or coupled cluster) form, Phys. Rep. 36, 1 (1978).

[72] R. F. Bishop and K. H. Lührmann, Electron correlations: I. Ground-state results in the high-density regime, Phys. Rev. B 17, 3757 (1978).

[73] R. F. Bishop and K. H. Lührmann, Electron correlations. II. Ground-state results at low and metallic densities, Phys. Rev. B 26, 5523 (1982).

[74] J. Arponen, Variational principles and linked-cluster exp $S$ expansions for static and dynamic many-body problems, Ann. Phys. (NY) 151, 311 (1983).

[75] R. F. Bishop and H. G. Kümmel, The coupled-cluster method, Phys. Today 40(3), 52 (1987).

[76] J. S. Arponen, R. F. Bishop, and E. Pajanne, Extended coupled-cluster method. I. Generalized coherent bosonization as a mapping of quantum theory into classical Hamiltonian mechanics, Phys. Rev. A 36, 2519 (1987).

[77] J. S. Arponen, R. F. Bishop, and E. Pajanne, Extended coupled-cluster method. II. Excited states and generalized random-phase approximation, Phys. Rev. A 36, 2539 (1987).

[78] R. J. Bartlett, Coupled-cluster approach to molecular structure and spectra: A step toward predictive quantum chemistry, J. Phys. Chem. 93, 1697 (1989).

[79] J. S. Arponen and R. F. Bishop, Independent-cluster parametrizations of wave functions in model field theories. I. Introduction to their holomorphic representations, Ann. Phys. (NY) 207, 171 (1991).

[80] R. F. Bishop, An overview of coupled cluster theory and its applications in physics, Theor. Chim. Acta 80, 95 (1991).

[81] R. F. Bishop, The coupled cluster method, in Microscopic Quantum Many-Body Theories and Their Applications, 
Lecture Notes in Physics Vol. 510, edited by J. Navarro and A. Polls (Springer, Berlin, 1998), pp. 1-70.

[82] C. Zeng, D. J. J. Farnell, and R. F. Bishop, An efficient implementation of high-order coupled-cluster techniques applied to quantum magnets, J. Stat. Phys. 90, 327 (1998).

[83] D. J. J. Farnell and R. F. Bishop, The coupled cluster method applied to quantum magnetism, in Quantum Magnetism, Lecture Notes in Physics Vol. 645, edited by Ulrich Schollwöck, Johannes Richter, Damian J. J. Farnell, and Raymond F. Bishop (Springer, Berlin, 2004), pp. 307-348.

[84] R. J. Bartlett and M. Musiał, Coupled-cluster theory in quantum chemistry, Rev. Mod. Phys. 79, 291 (2007).

[85] R. F. Bishop, P. H. Y. Li, and C. E. Campbell, Highly frustrated spin-lattice models of magnetism and their quantum phase transitions: A microscopic treatment via the coupled cluster method, AIP Conf. Proc. 1620, 40 (2014).

[86] K. Hida, Low temperature properties of the double layer quantum Heisenberg antiferromagnet: Modified spin wave method, J. Phys. Soc. Jpn. 59, 2230 (1990).

[87] K. Hida, Quantum disordered state without frustration in the double layer Heisenberg antiferromagnet: Dimer expansion and projector Monte Carlo study, J. Phys. Soc. Jpn. 61, 1013 (1992).

[88] A. J. Millis and H. Monien, Spin Gaps and Spin Dynamics in $\mathrm{La}_{2-x} \mathrm{Sr}_{x} \mathrm{CuO}_{4}$ and $\mathrm{YBa}_{2} \mathrm{Cu}_{3} \mathrm{O}_{7-\delta}$, Phys. Rev. Lett. 70, 2810 (1993).

[89] A. J. Millis and H. Monien, Spin gaps and bilayer coupling in $\mathrm{YBa}_{2} \mathrm{Cu}_{3} \mathrm{O}_{7-\delta}$ and $\mathrm{YBa}_{2} \mathrm{Cu}_{4} \mathrm{O}_{\delta}$, Phys. Rev. B 50, 16606 (1994).

[90] A. W. Sandvik and D. J. Scalapino, Order-Disorder Transition in a Two-Layer Quantum Antiferromagnet, Phys. Rev. Lett. 72, 2777 (1994).

[91] A. W. Sandvik, A. V. Chubukov, and S. Sachdev, Quantum critical behavior in a two-layer antiferromagnet, Phys. Rev. B 51, 16483(R) (1995).

[92] A. V. Chubukov and D. K. Morr, Phase transition, longitudinal spin fluctuations, and scaling in a two layer antiferromagnet, Phys. Rev. B 52, 3521 (1995).

[93] Z. Weihong, Various series expansions for the bilayer $S=\frac{1}{2}$ Heisenberg antiferromagnet, Phys. Rev. B 55, 12267 (1997).

[94] P. V. Shevchenko and O. P. Sushkov, Brueckner approach to the spin-wave gap critical index for the two-layer Heisenberg antiferromagnet, Phys. Rev. B 59, 8383 (1999).

[95] P. V. Shevchenko, A. W. Sandvik, and O. P. Sushkov, Double-layer Heisenberg antiferromagnet at finite temperature: Brueckner theory and quantum Monte Carlo simulations, Phys. Rev. B 61, 3475 (2000).

[96] L. Wang, K. S. D. Beach, and A. W. Sandvik, High-precision finite-size scaling analysis of the quantum-critical point of $S=$ 1/2 Heisenberg antiferromagnetic bilayers, Phys. Rev. B 73, 014431 (2006).

[97] A. Collins and C. J. Hamer, Two-particle bound states and oneparticle structure factor in a Heisenberg bilayer system, Phys. Rev. B 78, 054419 (2008).

[98] L. Fritz, R. L. Doretto, S. Wessel, S. Wenzel, S. Burdin, and M. Vojta, Cubic interactions and quantum criticality in dimerized antiferromagnets, Phys. Rev. B 83, 174416 (2011).
[99] R. Ganesh, S. V. Isakov, and A. Paramekanti, Néel to dimer transition in spin- $S$ antiferromagnets: Comparing bond operator theory with quantum Monte Carlo simulations for bilayer Heisenberg models, Phys. Rev. B 84, 214412 (2011).

[100] J. Helmes and S. Wessel, Entanglement entropy scaling in the bilayer Heisenberg spin system, Phys. Rev. B 89, 245120 (2014).

[101] T. Devakul and R. R. P. Singh, Quantum critical universality and singular corner entanglement entropy of bilayer Heisenberg-Ising model, Phys. Rev. B 90, 064424 (2014).

[102] M. Lohöfer, T. Coletta, D. G. Joshi, F. F. Assaad, M. Vojta, S. Wessel, and F. Mila, Dynamical structure factors and excitation modes of the bilayer Heisenberg model, Phys. Rev. B 92, 245137 (2015).

[103] K. Hida, Modified spin wave theory of the bilayer square lattice frustrated quantum Heisenberg antiferromagnet, J. Phys. Soc. Jpn. 65, 594 (1996).

[104] K. Hida, Dimer expansion study of the bilayer square lattice frustrated quantum Heisenberg antiferromagnet, J. Phys. Soc. Jpn. 67, 1540 (1998).

[105] J. Stapmanns, P. Corboz, F. Mila, A. Honecker, B. Normand, and S. Wessel, Thermal Critical Points and Quantum Critical End Point in the Frustrated Bilayer Heisenberg Antiferromagnet, Phys. Rev. Lett. 121, 127201 (2018).

[106] F. Alet, K. Damle, and S. Pujari, Sign-Problem-Free Monte Carlo Simulation of Certain Frustrated Quantum Magnets, Phys. Rev. Lett. 117, 197203 (2016).

[107] R. F. Bishop, P. H. Y. Li, R. Darradi, and J. Richter, The quantum $J_{1}-J_{1}^{\prime}-J_{2}$ spin-1 Heisenberg model: Influence of the interchain coupling on the ground-state magnetic ordering in 2D, Europhys. Lett. 83, 47004 (2008).

[108] R. F. Bishop, P. H. Y. Li, R. Darradi, J. Richter, and C. E. Campbell, The effect of anisotropy on the ground-state magnetic ordering of the spin-1 quantum $J_{1}^{X X Z}-J_{2}^{X X Z}$ model on the square lattice, J. Phys.: Condens. Matter 20, 415213 (2008).

[109] R. Haghshenas, W.-W. Lan, S.-S. Gong, and D. N. Sheng, Quantum phase diagram of spin-1 $J_{1}-J_{2}$ Heisenberg model on the square lattice: An infinite projected entangled-pair state and density matrix renormalization group study, Phys. Rev. B 97, 184436 (2018).

[110] H. C. Jiang, F. Krüger, J. E. Moore, D. N. Sheng, J. Zaanen, and Z. Y. Weng, Phase diagram of the frustrated spatiallyanisotropic $S=1$ antiferromagnet on a square lattice, Phys. Rev. B 79, 174409 (2009).

[111] P. H. Y. Li and R. F. Bishop, Collinear antiferromagnetic phases of a frustrated spin- $\frac{1}{2} J_{1}-J_{2}-J_{1}^{\perp}$ Heisenberg model on an $A A$-stacked bilayer honeycomb lattice, J. Magn. Magn. Mater. 482, 262 (2019).

[112] We use the program package CCCM of D. J. J. Farnell and J. Schulenburg, see http://www-e.uni-magdeburg.de/jschulen/ ccm/index.html

[113] D. J. J. Farnell, O. Götze, J. Schulenburg, R. Zinke, R. F. Bishop, and P. H. Y. Li, Interplay between lattice topology, frustration, and spin quantum number in quantum antiferromagnets on Archimedean lattices, Phys. Rev. B 98, 224402 (2018). 